Números de Lê e classes de Milnor de hipersuperfícies analíticas complexas

\author{
Michelle Ferreira Zanchetta
}




\title{
Números de Lê e classes de Milnor de hipersuperfícies analíticas complexas
}

\author{
Michelle Ferreira Zanchetta \\ Orientador: Prof. Dr. Marcelo José Saia
}

Co-Orientador: Prof. Dr. José Antonio Seade Kuri

Tese apresentada ao Instituto de Ciências Matemáticas e de Computação - ICMC-USP, como parte dos requisitos para obtenção do título de Doutor em Matemática.

USP - São Carlos

janeiro / 2010 
À minha família e ao meu noivo, Leandro. 


\section{Agradecimentos}

Primeiramente a Deus, por ter me sustentado por todo esse caminho.

Aos meus queridos pais, Luiz e Etelvina, pelo constante apoio e incentivo. Mãe, obrigada por suas orações, que foram fundamentais para me fortalecer e me guiar em minhas escolhas.

Aos meus irmãos, Gustavo e Gabriela, pela amizade e pelo carinho.

Ao meu noivo Leandro, por ter me apoiado com muito amor, paciência e companheirismo, pelas alegrias somadas e problemas compartilhados, por confiar no meu caráter, na minha competência e no nosso futuro. Obrigada por estar na minha vida.

Ao Prof. Marcelo José Saia, pela orientação, dedicação, incentivo, confiança e, principalmente, pela amizade. Obrigada, pois aprendi muito nestes anos juntos.

Ao Prof. José Seade, que também tive o privilégio de trabalhar. Foi uma honra e um prazer trabalhar com esse grande nome da matemática. Obrigada pela acolhida e disposição que me dedicou quando estive em Cuernavaca, pela paciência e atenção em responder as minhas dúvidas, sem falar nos conhecimentos matemáticos que adquiri.

A todos os professores que contribuíram para a minha chegada até aqui, em especial, ao Prof. Roberto Callejas Bedregal que por várias vezes se dispôs a responder minhas dúvidas e com quem aprendi muita matemática.

A todos meus amigos do ICMC, que de uma forma ou de outra, me ajudaram nesta caminhada. Em especial ao Aurélio, que foi meu grande amigo e companheiro quando estive no México.

Aos funcionários do ICMC/USP e do Instituto de Matemáticas da UNAM em Cuernavaca.

À CAPES e ao CNPq pelo suporte financeiro.

Enfim, a todos que colaboraram para a concretização deste trabalho. 


\section{Resumo}

Este trabalho está dividido em duas partes distintas.

Na primeira parte caracterizamos os números de Lê de polinômios que são produtos de polinômios de Pham-Brieskorn de mesmo tipo, que denominamos de arranjos de Pham-Brieskorn, obtendo fórmulas para estes números somente utilizando o número de variáveis, os pesos e o grau de homogeneidade destes polinômios.

Na segunda parte nos dedicamos a estabelecer relações entre os números de Lê, que é um conceito local, e as classes de Milnor, que são objetos globais que fornecem informações quanto a geometria e topologia de hipersuperfícies analíticas complexas. No contexto geral, usando a hipótese de especialização, relacionamos a classe de Milnor de dimensão máxima de uma hipersuperfície $Z$ numa variedade compacta $M$ com uma soma, sobre os estratos de uma estratificação de Whitney de $Z$ (com estratos conexos) que estão contidos no conjunto singular, em termos do último número de Lê associado a cada estrato. Além disso, obtivemos uma caracterização da classe de Milnor de dimensão mínima via os números de Lê sem usar a hipótese de especialização. Esta classe coincide com o chamado número de Milnor de Parusinski que, assim como os números de Lê, também é uma generalização do número de Milnor. 


\section{Abstract}

This work is divided into two distinct parts.

In the first part we characterize the Lê numbers of polynomials that are products of PhamBrieskorn polynomials of the same type that we call Pham-Brieskorn arrangements, obtaining formulas to these numbers only using the number of variables, weights and degree of homogeneity of these polynomials.

In the second part we are dedicated to establishing relationships between Lê numbers, which is a local concept, and the Milnor classes, which are global objects that provide information about the geometry and topology of complex analytic hypersurfaces. In a general context, using the hypothesis of specialization we relate the top dimensional Milnor class of a hypersurface $Z$ in a compact manifold $M$ with a sum given in terms of the last Lê number associated to each stratum of a Whitney estratification of $Z$ (with connected strata) that are contained in singular set. Moreover, we obtain a characterization of the Milnor class of minimum dimension via the Lê numbers without using the hypothesis of specialization. This class coincides with the Milnor number of Parusinski that, as the Lê numbers, it is also a generalization of the Milnor number. 


\section{Sumário}

1 Preliminares 4

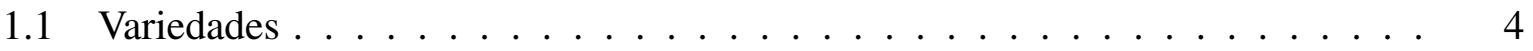

1.1.1 Variedades suaves .................... 4

1.1.2 Variedades complexas ................ 5

1.1.3 Variedades analíticas ................... 6

1.2 Orientação e grau de uma aplicação . . . . . . . . . . . . . . . . . . 6

1.3 Feixes e espaços analíticos complexos . . . . . . . . . . . . 7

1.3.1 Espaço analítico complexo de um feixe lacuna . . . . . . . . . . . 10

1.4 Ciclos analíticos . . . . . . . . . . . . . . . . . . 11

1.5 Fibrado vetorial complexo . . . . . . . . . . . . . . . . . 13

1.6 Variedades polares - definidas por Lê e Teissier . . . . . . . . . . . . . . 14

1.6.1 Multiplicidades polares . . . . . . . . . . . . . 15

1.7 Estratificação de Whitney . . . . . . . . . . . . . . . . . . . 15

1.7.1 Estratificação de Whitney minimal - descrita por Lê e Teissier . . . . . 17

1.8 Homologia e cohomologia . . . . . . . . . . . . . . . 17

1.8.1 Homologia (cohomologia) simplicial . . . . . . . . . . . . . . 18

1.9 Fibração de Milnor . . . . . . . . . . . . . . . . . . . . . . . . . . . 20

2 Números de Lê

2.1 Números de Lê de germes de funções holomorfas . . . . . . . . . . . . . . . . 22

2.1.1 Coordenadas pré-polares . . . . . . . . . . . . . . 25

2.2 Números de Lê de hipersuperfícies . . . . . . . . . . . . . . . . . . . . 26 
3 Arranjos de Pham-Brieskorn 28

3.1 Raio polar e fórmulas de Lê-Iomdine generalizadas . . . . . . . . . . . . . . 29

3.2 Coordenadas pré-polares para arranjos de Pham-Brieskorn . . . . . . . . . 30

3.3 Raio polar de Arranjos de Pham-Brieskorn . . . . . . . . . . . . . . . . . 33

3.4 Caracterização dos números de Lề . . . . . . . . . . . . . . . . . . . . . . . 37

4 Classes Características para Variedades Singulares 42

4.1 Classes de Chern de um fibrado . . . . . . . . . . . . . . . . . 42

4.2 Classes de Schwartz-MacPherson . . . . . . . . . . . . . . . . . 44

4.2.1 Conjectura de Deligne-Grothendieck . . . . . . . . . . . . 44

4.2.2 Blow-up de Nash . . . . . . . . . . . . . . . . 45

4.2 .3 Obstrução local de Euler . . . . . . . . . . . . . . . . . . . . 46

4.2.4 Classe de Mather e o isomorfismo T . . . . . . . . . . . . 47

4.3 Classes de Fulton-Johnson . . . . . . . . . . . . . . . . . . . . . . 49

5 Classes de Milnor $\quad 50$

5.1 Classe de Milnor de dimensão máxima . . . . . . . . . . . . . . . . . . . . 53

5.2 Classe de Milnor de dimensão mínima . . . . . . . . . . . . . . . . . . 55

$\begin{array}{ll}\text { Bibliografia } & 58\end{array}$ 


\section{Introdução}

No estudo de variedades analíticas complexas singulares, existe uma rica interação entre álgebra e análise com geometria e topologia. Para o caso de pontos críticos de funções holomorfas com um ponto crítico isolado, John Milnor introduziu em [19] um importante invariante, agora chamado número de Milnor, que tem interpretações em termo de álgebra, topologia, geometria diferencial e assim por diante. Esse invariante tem um importante papel na teoria moderna de singularidades e tem sido generalizado em muitas direções. Uma importante generalização do número de Milnor foi dada por David Massey em [17], onde ele introduziu os números de Lê e os ciclos de Lê associados à funções holomorfas com pontos críticos não isolados, ou equivalentemente, à singularidades não isoladas em hipersuperfícies analíticas complexas. A definição destes números é analítica mas eles também podem ser interpretados de maneira topológica ([17], Teorema 3.3), em geometria algébrica ([9], 2.1), entre outras.

Sob o aspecto analítico, Massey descreve, em [17] no Teorema 4.5, as chamadas fórmulas de Lê-Iomdine generalizadas que é uma técnica que reduz uma hipersuperfície com conjunto singular de dimensão arbitrária $s$ a uma hipersuperfície com conjunto singular de dimensão $s-1$ e relaciona os números de Lê da primeira com os números de Lê desta hipersuperfície obtida. Massey utiliza estas relações para obter uma fórmula, que é um tipo de fórmula de Plücker, envolvendo os números de Lê de um polinômio homogêneo de grau $d$, somente utilizando o grau e o número de variáveis ([17], Corolário 4.7). Com o auxílio desta fórmula, ele caracteriza cada número de Lê de um arranjo de hiperplanos, ou seja, produto de funções lineares.

Na primeira parte da tese, utilizando esta técnica e outras ferramentas de Teoria de Singularidades, obtivemos, no caso de um polinômio que é produto de polinômios de Pham-Brieskorn do mesmo tipo, uma relação semelhante à de Massey dos números de Lê deste polinômio somente utilizando o número de variáveis, os pesos e o grau de homogeneidade e consequente- 
mente caracterizamos seus números de Lê. A este tipo de polinômio denominamos de arranjo de Pham-Brieskorn.

Por outro lado, no intuito de obter informações quanto a Geometria e a Topologia de hipersuperfícies analíticas complexas aparecem as classes características para variedades singulares. Elas surgiram para generalizar as classes de Chern, que são classes características de variedades complexas suaves definidas inicialmente como obstrução à construção de campos de $k$-referenciais tangentes. Existem pelo menos três maneiras de obter esta generalização. A primeira consiste em considerar a união dos fibrados tangentes aos estratos de uma estratificação da variedade e considerar a obstrução à construção de campos de $k$-referenciais adaptados, introduzido por M.H. Schwartz em [26]. A segunda foi definida por R. MacPherson em [16], que utiliza o fibrado de Nash generalizando no sentido axiomático as classes de Chern com uma prova da conjectura de Deligne e Grothendieck sobre a existência e unicidade de classes características de variedades singulares. J.P. Brasselet e M.-H. Schwartz mostram em [2], via o isomorfismo de Alexander, que as definições de M.H. Schwartz e de R. MacPherson são as mesmas e desde então estas são chamadas classes de Schwartz-MacPherson. Uma terceira maneira de generalizar as classes de Chern foi introduzida por Fulton e Johnson em [8] onde eles utilizam o fibrado virtual em substituição ao fibrado tangente, que em geral são distintas das classes de SchwartzMacPherson. Esta diferença tem sido investigada por vários pesquisadores e é chamada de Classe de Milnor.

Considerando $Z$ uma hipersuperfície contida em uma variedade suave complexa compacta, temos que as classes de Milnor de $Z$ tem suporte no conjunto singular de $Z$. Assim, no caso de singularidades isoladas, somente a classe de Milnor de dimensão 0 é não nula e é obtida como a soma dos números de Milnor nos pontos singulares. Por outro lado, dada uma estratificação de Whitney com estratos conexos de $Z$, os números de Lê genéricos associados (que são definidos em cada ponto da hipersuperfície) são constantes ao longo de cada estrato. Com isso, surge o interesse de saber se podemos relacionar as classes de Milnor de $Z$ com os números de Lê associados a cada estrato desta estratificação.

Usando a descrição das classes de Milnor via especialização dada por Parusinski e Pragacz em [25], expressamos a classe de Milnor de dimensão máxima como uma soma, sobre os estratos de uma estratificação de Whitney (com estratos conexos) que estão contidos no conjunto singu- 
lar, em termos do número de Milnor transversal de cada estrato. Esta é uma generalização de um resultado conhecido de Brasselet, Lehmann, Seade e Suwa [4] que é válido somente quando o conjunto singular é não singular. Além disso, expressamos o número de Milnor transversal como um número de Lê.

Agora, sem usar a hipótese de especialização, obtivemos uma descrição da classe de Milnor de dimensão mínima via os números de Lê. Ressaltamos aqui a importância deste resultado pois este fornece uma interpretação geométrica do número de Milnor de Parusinski, que também é uma generalização do número de Milnor. Usamos o fato de que a classe de Milnor de dimensão mínima coincide com o número de Milnor de Parusinski.

A distribuição desta tese é feita da seguinte maneira:

O capítulo 1 contém as definiçõos e os resultados preliminares gerais que são necessários ao longo deste trabalho.

No capítulo 2 é dada uma atenção especial para os conceitos que envolvem os números de Lê, devido a sua importância.

O capítulo 3 é dedicado aos arranjos de Pham-Brieskorn, onde é feita a caracterização de seus números de Lê utilizando somente o número de variáveis, os pesos e o grau de homogeneidade destes arranjos.

Apesar de as classes características para variedades singulares serem pré-requisitos, sua importância na definição das classes de Milnor mereceu um lugar de destaque e dedicamos o capítulo 4 para definir tais conceitos.

No capítulo 5 são estudadas as classes de Milnor de uma hipersuperfíce em uma variedade compacta e, usando a hipótese de especialização, caracterizamos a classe de Milnor de dimensão máxima em função do último número de Lê associado a cada estrato de uma estratificação de Whitney desta hipersuperfície. Neste capítulo também demonstramos, através de um ponto de vista diferente, o resultado já conhecido que as classes de Milnor têm suporte no conjunto singular. Para isto usamos a caracterização das classes de Milnor dada por Parusinski e Pragacz em [25]. Para finalizar descrevemos um dos mais importantes resultados deste trabalho, que é a descrição da classe de Milnor de dimensão mínima via os números de Lê pois com isto demos uma interpretação geométrica ao número de Milnor de Parusinski. Ressaltamos que neste caso não foi necessário o uso da hipótese de especialização. 


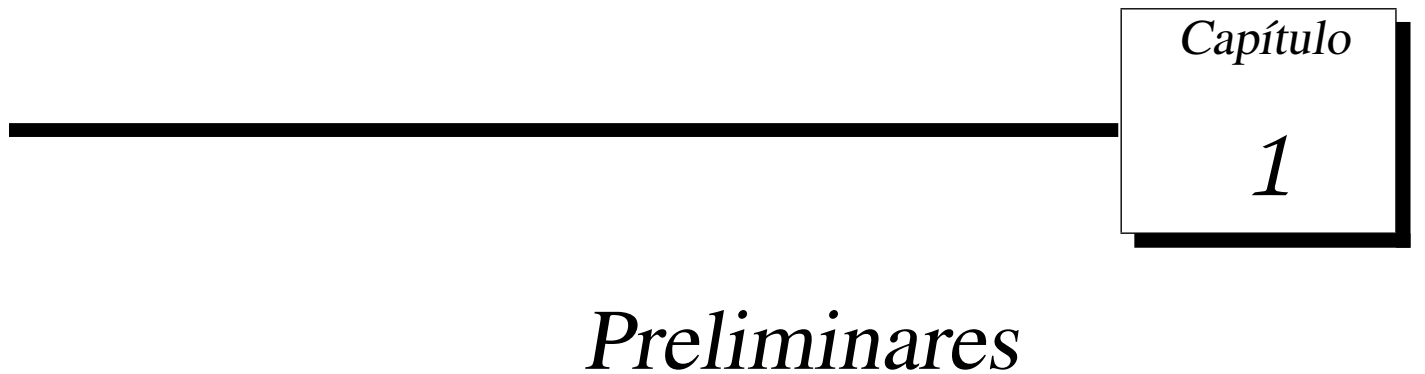

O objetivo deste capítulo é fixar notações utilizadas no texto e dar noções básicas necessárias para o entendimento dos conceitos que são usados ao longo deste trabalho. Referimo-nos sempre às referências para maiores detalhes e demonstrações.

\section{$1.1 \quad$ Variedades}

Nesta seção especificamos cada conceito de variedade que foi utilizado neste trabalho e introduzimos o conceito de germe. As referências para detalhes são [14], [12] e [10].

\subsubsection{Variedades suaves}

Definição 1.1. Seja $M$ um espaço topológico de Hausdorff. Dizemos que $M$ é uma variedade suave de dimensão $n$ se

1) existe uma família de conjuntos abertos $\left(U_{\alpha}\right)_{\alpha \in I}$ tal que $\bigcup_{\alpha \in I} U_{\alpha}=M$,

2) para cada $\alpha \in I$ existe um homeomorfismo $\phi_{\alpha}: U_{\alpha} \rightarrow \phi_{\alpha}\left(U_{\alpha}\right) \subset \mathbb{R}^{n}\left(\phi_{\alpha}^{-1}\right.$ é chamada de parametrização de $M)$,

3) para $\alpha, \beta \in I$, $\phi_{\alpha} \circ \phi_{\beta}^{-1}$ (nos seus domínios de definição) é uma função suave em $\mathbb{R}^{n}$.

Sejam $\left(M,\left\{\phi_{\alpha}\right\}\right)$ e $\left(N,\left\{\psi_{\beta}\right\}\right)$ variedades e $f: M \rightarrow N$ uma aplicação contínua. Dizemos que $f$ é diferenciável (suave) se para cada $x \in M$, existam abertos $U_{\alpha}$ e $V_{\beta}$ contendo $x$ e $f(x)$ respectivamente com $f\left(U_{\alpha}\right) \subseteq V_{\beta}$ tal que $\psi_{\beta} \circ f \circ \phi_{\alpha}^{-1}: \phi_{\alpha}\left(U_{\alpha}\right) \rightarrow \psi_{\beta}\left(V_{\beta}\right)$ é diferenciável (suave).

Para cada $x \in M$, definimos o espaço tangente a $M$ em $x, T_{x} M$, como sendo

$$
T_{x} M:=\left\{v=\gamma^{\prime}(0) \mid \gamma:\right]-\varepsilon, \varepsilon[\rightarrow M \text { diferenciável com } \gamma(0)=x\} .
$$


Se $f$ é diferenciável (suave), definimos a derivada de $f$ em $x \in M$ como sendo a aplicação $d_{x} f: T_{x} M \rightarrow T_{f(x)} N$ definida por $d_{x} f(v)=(f \circ \gamma)^{\prime}(0)$ para $v=\gamma^{\prime}(0)$.

Seja $f: U \rightarrow \mathbb{C}$ uma função definida em um conjunto aberto $U \subseteq \mathbb{C}$. Dizemos que $f$ é holomorfa se satisfaz as equações de Cauchy-Riemann, ou seja, se escrevemos $f(z)=u(z)+$ $i v(z) \operatorname{com} z=x+i y$, temos que

$$
\frac{\partial u}{\partial x}-\frac{\partial v}{\partial y}=0 \text { e } \frac{\partial u}{\partial y}+\frac{\partial v}{\partial x}=0
$$

Sejam $N$ e $P$ variedades suaves de dimensões $n$ e $p$ respectivamente, e $x \in N$. No conjunto das aplicações holomorfas definidas numa vizinhança de $x$ em $N$ e com valores em $P$, introduzimos a seguinte relação de equivalência:

Definição 1.2. Duas aplicações holomorfas $f_{1}: U_{1} \rightarrow P$ e $f_{2}: U_{2} \rightarrow P$ são equivalentes quando existir uma vizinhança $U \subset U_{1} \cap U_{2}$ de $x$ em $N$ tal que as restrições $\left.f_{1}\right|_{U}$ e $\left.f_{2}\right|_{U}$ coincidem.

As classes de equivalência dessa relação são chamadas de germes de aplicações em $x$ e um elemento da classe de equivalência é chamado de representante do germe, que denotamos da forma $f:(N, x) \rightarrow(P, y), f(x)=y$.

Se $N=\mathbb{C}^{n}$ e $P=\mathbb{C}^{p}$, denotamos por $\mathscr{O}_{n, p}$ o conjunto dos germes na origem das aplicações holomorfas $f:\left(\mathbb{C}^{n}, 0\right) \rightarrow \mathbb{C}^{p}$ onde $f(0)$ não é necessariamente 0 . Quando $p=1$, denotamos este conjunto por $\mathscr{O}_{n}$, que é um anel local cujo ideal maximal $\mathscr{M}_{n}=\left\{f \in \mathscr{O}_{n} \mid f(0)=0\right\}$.

\subsubsection{Variedades complexas}

Definição 1.3. Seja $M$ um espaço topológico Hausdorff. Dizemos que $M$ é uma variedade complexa $n$-dimensional se ela admite uma cobertura aberta $\mathscr{U}=\left\{U_{\alpha}\right\}_{\alpha \in I}$ com as seguintes propriedades:

1. Para cada $\alpha$, existe um homeomorfismo $\psi_{\alpha}$ de $U_{\alpha}$ em um aberto $D_{\alpha}$ em $\mathbb{C}^{n}$.

2. Para cada par $(\alpha, \beta)$, a aplicação $\psi_{\alpha} \circ \psi_{\beta}^{-1}$ é biholomorfa (holomorfa, bijetora e com inversa holomorfa $)$ de $\psi_{\beta}\left(U_{\alpha} \cup U_{\beta}\right)$ em $\psi_{\alpha}\left(U_{\alpha} \cup U_{\beta}\right)$.

Observação 1.4. Uma variedade complexa pode ser vista como uma variedade suave. Claramente a recíproca não é verdadeira. 
Uma aplicação $f: M \rightarrow N$ entre variedades complexas é chamada holomorfa se ela é dada em termos de coordenadas holomorfas locais em $N$ por funções holomorfas.

\subsubsection{Variedades analíticas}

Definição 1.5. Dizemos que um subconjunto $V$ de um conjunto aberto $U \subseteq \mathbb{C}^{n}$ é uma variedade analítica (afim) em $U$ se, para todo $p \in U$, existe uma vizinhança $U^{\prime}$ de p em $U$ tal que $V \cap U^{\prime}$ é o conjunto de zeros comuns de uma coleção finita de funções holomorfas $f_{1}, \cdots, f_{k}$ em $U^{\prime}$.

Em particular, $V$ é chamado de hipersuperfície analítica (afim) se $V$ é localmente o conjunto de zeros de uma única função holomorfa. Quando as funções $f_{1}, \cdots, f_{k}$ são polinomiais dizemos que $V$ é uma variedade algébrica (afim).

Uma variedade analítica $V$ é redutível se pode ser escrita como $V=V_{1} \cup V_{2}$, onde $V_{i}, i=1,2$ são variedades analíticas. Caso contrário, dizemos que $V$ é irredutível.

Considere os pares $\left(X_{\alpha}, U_{\alpha}\right)$, onde $U_{\alpha}$ é uma vizinhança da origem em $\mathbb{C}^{n}$ e $X_{\alpha}$ é uma variedade analítica (algébrica) de $U_{\alpha}$.

Definição 1.6. Dois pares $\left(X_{1}, U_{1}\right) e\left(X_{2}, U_{2}\right)$ são equivalentes se existe uma vizinhança $W \subset$ $U_{1} \cap U_{2}$ da origem tal que $W \cap X_{1}=W \cap X_{2}$.

Isto define uma relação de equivalência. Uma classe de equivalência destes pares é chamada de germe de uma variedade analítica (algébrica) na origem em $\mathbb{C}^{n}$.

Analogamente ao caso de variedades analíticas em $\mathbb{C}^{n}$, podemos definir variedade analítica em uma variedade complexa.

Definição 1.7. Uma subvariedade analítica $V$ de uma variedade complexa $M$, também chamada de variedade analítica complexa, é um subconjunto de $M$ dado localmente como zeros de uma coleção $f_{1}, \cdots, f_{k}$ de funções holomorfas.

\subsection{Orientação e grau de uma aplicação}

A referência para detalhes é [20].

Inicialmente vamos definir o conceito de orientação em uma variedade suave e em seguida dizer quando uma aplicação entre variedades suaves orientadas de mesma dimensão preserva ou não a orientação. Isto é feito a nível de espaços vetoriais. 
Definição 1.8. Dar uma orientação a um espaço vetorial é definir a seguinte relação de equivalência entre suas bases:

Sejam $A$ e $B$ duas bases de um espaço vetorial $V$. Dizemos que A é equivalente a $B$, se a matriz mudança de base de A para B tiver determinante positivo.

Definição 1.9. Dar uma orientação a uma variedade suave $M$ é escolher orientações para os espaços tangentes satisfazendo: para cada $x \in M$ existe uma parametrização $h: U \rightarrow M$ tal que $d_{u} h: \mathbb{R}^{m} \rightarrow T_{x} M$ preserva a orientação para todo $u \in U$.

Seja $f: M \rightarrow N$ uma aplicação suave entre variedades suaves de mesma dimensão $m$. Dizemos que $x \in M$ é um ponto regular de $f$ se a diferencial $d_{x} f: T_{x} M \rightarrow T_{f(x)} N$ tem posto máximo e $y \in N$ é chamado de valor regular de $f$ se $f^{-1}(y)$ possuir somente pontos regulares de $f$.

Agora, dada $f: M \rightarrow N$ uma aplicação suave entre variedades suaves de mesma dimensão $m$ com $M$ compacta e $N$ conexa, sabemos que, para cada ponto regular $x \in M, d_{x} f: T_{x} M \rightarrow T_{f(x)} N$ é um isomorfismo, que pode preservar a orientação ou revertê-la. No primeiro caso, colocamos sign $d_{x} f=+1$, e no segundo caso, colocamos sign $d_{x} f=-1$.

Se $M$ é compacta e $y \in N$ é um valor regular, pode-se mostrar que $f^{-1}(p)$ é vazio ou finito.

Definição 1.10. Para cada valor regular $y \in N$ definimos $o$ grau de $f$ em y como

$$
\operatorname{grau}(f)=\sum_{x \in f^{-1}(y)} \operatorname{sign} d_{x} f
$$

É possível mostrar que o grau de $f$ independe do valor regular escolhido e, como pelo Lema de Sard o conjunto dos pontos regulares de $f$ é um conjunto denso em $N$, temos a seguinte definição:

Definição 1.11. O grau de $f$, denotado por grau $(f)$, é definido como sendo o grau de $f$ em um ponto genérico de $N$.

Observação 1.12. No caso de variedades complexas temos que $d_{x} f$ sempre preserva a orientação. Assim, o grau $(f)=\# f^{-1}(y)$, com y um ponto genérico de $N$.

\subsection{Feixes e espaços analíticos complexos}

As referências para detalhes são [6] e [1]. 
Definição 1.13. Seja X um espaço topológico. Um feixe $\mathscr{F}$ de anéis (respectivamente, módulos) sobre $X$, consiste do seguinte:

1) Para cada aberto $U$ de $X$, existe um anel (módulo) $\Gamma(U, \mathscr{F})$ chamado conjunto de seções de $\mathscr{F}$ sobre $U$.

2) Para cada par de abertos $V \subseteq U$ de $X$, existe uma aplicação de restrição

$$
r_{U, V}: \Gamma(U, \mathscr{F}) \rightarrow \Gamma(V, \mathscr{F}), r_{U, V}(s)=s_{\left.\right|_{V}}
$$

satisfazendo:

i) $r_{U, U}=i d_{\Gamma(U, \mathscr{F})}$ para cada aberto $U$ de $X$;

ii) $r_{V, W} \circ r_{U, V}=r_{U, W}$ para cada $W \subseteq V \subseteq U$ abertos de $X$.

3) Para cada aberto $U$ de $X$ e cada cobertura de $U$ por abertos $U=\cup_{i \in I} U_{i}$, de $X$ tem-se :

i) para quaisquer $s_{1}, s_{2} \in \Gamma(U, \mathscr{F})$, se $s_{\left.\right|_{U_{i}}}=\left.s_{2}\right|_{U_{i}} \forall i \in I$, então $s_{1}=s_{2}$;

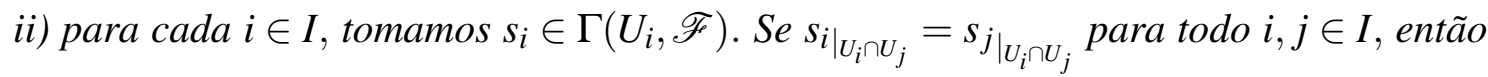
existe $s \in \Gamma(U, \mathscr{F})$ tal que $s_{\left.\right|_{i}}=s_{i}$ para todo $i \in I$.

Exemplo 1.14. Em $\mathbb{C}^{n}$, considerando a topologia usual, definimos o feixe $\mathscr{O}_{\mathbb{C}^{n}}$ da seguinte maneira: dado $U \subseteq \mathbb{C}^{n}$ aberto, $\mathscr{O}_{\mathbb{C}^{n}}(U):=\{f: U \rightarrow \mathbb{C} \mid f$ holomorfa $\}$. Para $V \subseteq U$ abertos, consideremos as restrições naturais $\rho_{V}^{U}: \mathscr{O}_{\mathbb{C}^{n}}(U) \rightarrow \mathscr{O}_{\mathbb{C}^{n}}(V), \operatorname{com} \rho_{V}^{U}(s)=s_{\left.\right|_{V}}$.

É fácil verificar que $\mathscr{O}_{\mathbb{C}^{n}}$ é um feixe de anéis.

Definição 1.15. Seja $\mathscr{F}$ um feixe de anéis (módulos) sobre X. Definimos o talo de $\mathscr{F}$ em x como o anel (módulo) $\mathscr{F}_{x}:=\{(U, s): U$ é aberto de $X, x \in U$ e $s \in \Gamma(U, \mathscr{F})\}$ módulo a relação $:$ $(U, s) \sim(V, t) \Leftrightarrow \exists W$ aberto de $X$ contido em $U \cap V$ tal que $s_{\left.\right|_{W}}=t_{\left.\right|_{W}}$.

Ressaltamos que o talo de $\mathscr{O}_{\mathbb{C}^{n}}$ em 0 é o anel local de germes de funções holomorfas de $\mathbb{C}^{n}$ em 0 , ou seja, $\mathscr{O}_{\mathbb{C}^{n}, 0}=\mathscr{O}_{n}$.

Definição 1.16. Um morfismo $\alpha: \mathscr{F} \rightarrow \mathscr{G}$ entre dois feixes de X é uma coleção de aplicações

$$
\alpha(V): \Gamma(V, \mathscr{F}) \rightarrow \Gamma(V, \mathscr{G})
$$


para cada $V$ aberto de $X$ tal que o diagrama

$$
\begin{array}{ccc}
\Gamma(V, \mathscr{F}) & \stackrel{\alpha(V)}{\longrightarrow} & \Gamma(V, \mathscr{G}) \\
r_{V, U} \downarrow & & \downarrow \tilde{r}_{V, U} \\
\Gamma(U, \mathscr{F}) & \stackrel{\alpha(U)}{\longrightarrow} & \Gamma(U, \mathscr{G})
\end{array}
$$

comuta sempre que $U \subset V$ são abertos de $X$.

Um morfismo $\alpha: \mathscr{F} \rightarrow \mathscr{G}$ induz um homomorfismo natural $\alpha_{x}: \mathscr{F}_{x} \rightarrow \mathscr{G}_{x}$ para todo $x \in X$. O morfismo $\alpha$ é injetor (sobrejetor) se para todo $x \in X, \alpha_{x}$ é injetor (sobrejetor).

Definição 1.17. Seja $Z$ um subespaço topológico de X. Definimos $\mathscr{F}_{\mid Z}$ como sendo o feixe onde $\Gamma\left(V, \mathscr{F}_{\mid Z}\right):=\lim _{U \supseteq i(V)} \Gamma(U, \mathscr{F})$ (limite direto), onde $V$ é um aberto de $Z, U$ é um aberto de $X e$ ié a aplicação inclusão.

Definição 1.18. Seja $\psi: X \rightarrow Y$ contínua e $\mathscr{F}$ um feixe sobre $X$. Para cada aberto $U$ de $Y$ $\operatorname{definimos}\left(\psi_{*}(\mathscr{F})\right)(U)=\mathscr{F}\left(\psi^{-1}(U)\right)$.

Verifica-se que $\psi_{*}(\mathscr{F})$ é um feixe sobre $Y$.

Definição 1.19. Um espaço anelado é um $\operatorname{par}(X, \mathscr{F})$ onde $X$ é um espaço topológico e $\mathscr{F}$ é um feixe de anéis sobre $X$.

Seja $D \subseteq \mathbb{C}^{n}$ domínio com a topologia induzida. Para cada aberto $U$ de $D$ consideremos $\mathscr{O}_{D}(U):=\{f: U \rightarrow \mathbb{C} \mid f$ holomorfa $\}$. Considerando as restrições naturais temos que $\mathscr{O}_{D}$ é um feixe de anéis sobre $D$.

Definição 1.20. Um espaço analítico complexo é um espaço anelado $\left(X, \mathscr{O}_{X}\right)$ onde $\mathscr{O}_{X}$ é um feixe de $\mathbb{C}$ álgebra locais (isto é, $\mathscr{O}_{X, x}$ é uma $\mathbb{C}$-álgebra local, $\forall x \in X$ ), tal que para cada aberto $U \subseteq X,\left(U, \mathscr{O}_{\left.X\right|_{U}}\right)$ é isomorfo a $\left(D, \mathscr{O}_{D}\right)$ para algum domínio $D \subseteq \mathbb{C}^{n}$, isto é, existe um par $(\varphi, \widetilde{\varphi})$, tal que $\varphi: U \rightarrow D$ é homeomorfismo e $\widetilde{\varphi}: \mathscr{O}_{D} \rightarrow \varphi_{*}\left(\left.\mathscr{O}_{X}\right|_{U}\right)$ é isomorfismo de feixes.

No decorrer do texto, para simplificar a notação, denotamos por $\mathscr{O}_{U}$ o feixe $\mathscr{O}_{\left.X\right|_{U}}$, onde $U$ é um aberto de $X$.

Observação 1.21. $\left(\mathbb{C}^{n}, \mathscr{O}_{\mathbb{C}^{n}}\right)$ é um espaço analítico complexo. 
Definição 1.22. Sejam $\left(X, \mathscr{O}_{X}\right)$ e $\left(Y, \mathscr{O}_{Y}\right)$ espaços analíticos complexos. Um morfismo entre eles é um par $(\varphi, \widetilde{\varphi})$ tal que $\varphi: Y \rightarrow X$ é contínua e $\widetilde{\varphi}: \mathscr{O}_{X} \rightarrow \varphi_{*}\left(\mathscr{O}_{Y}\right)$ é morfismo de feixes.

Notação: $(\varphi, \widetilde{\varphi}):\left(Y, \mathscr{O}_{Y}\right) \rightarrow\left(X, \mathscr{O}_{X}\right)$.

Definição 1.23. Sejam $\left(X, \mathscr{O}_{X}\right) e\left(Y, \mathscr{O}_{Y}\right)$ espaços analíticos complexos. Uma imersão fechada é um morfismo $(\varphi, \widetilde{\varphi}):\left(Y, \mathscr{O}_{Y}\right) \rightarrow\left(X, \mathscr{O}_{X}\right)$ tal que $\varphi(Y) \subseteq X$ é fechado e $\widetilde{\varphi}$ é sobrejetora.

A esta imersão fechada associamos um "feixe" $\mathscr{I}_{Y}$ sobre $X$ da seguinte maneira:

$\mathscr{I}_{Y}:=\operatorname{Ker}(\widetilde{\varphi})$, isto é, $\mathscr{I}_{Y}(U):=\operatorname{Ker}(\widetilde{\varphi}(U))$, onde $\widetilde{\varphi}(U): \mathscr{O}_{X}(U) \rightarrow \varphi_{*}\left(\mathscr{O}_{Y}\right)(U)$.

Temos que $\mathscr{I}_{Y}(U)$ é um ideal de $\mathscr{O}_{X}(U), \forall U \subseteq X$ aberto. Dizemos, neste caso, que $\mathscr{I}_{Y}$ é um feixe de ideais.

Além disso, se $\mathscr{O}_{X}$ é um feixe de anéis Noetherianos então $\mathscr{I}_{Y}(U)$ é finitamente gerado, para todo aberto $U$ de $X$. Neste caso, dizemos que $\mathscr{I}_{Y}$ é um feixe coerente de ideais.

Durante todo o trabalho denotaremos o espaço analítico complexo $\left(X, \mathscr{O}_{X}\right)$ simplesmente por $X$, ficando subentendido seu feixe. Como componentes irredutíveis do espaço analítico complexo $X$ queremos dizer as componentes irredutíveis do espaço topológico $X$.

\subsubsection{Espaço analítico complexo de um feixe lacuna}

Para compreender a próxima definição, que é uma estrutura algébrica essencial à definição dos números de Lê, é preciso entender a relação entre germes de variedades analíticas definidas na origem em $\mathbb{C}^{n}$ e os ideais $\mathscr{O}_{n}$.

Dado um germe de variedade analítica $X$ definido na origem de $\mathbb{C}^{n}$, associamos a este o ideal $I(X)$ em $\mathscr{O}_{n}$ constituído dos germes $f \in \mathscr{O}_{n}$ tais que $f(x)=0, \forall x \in X$. Por outro lado, para cada ideal $I$ de $\mathscr{O}_{n}, I=<f_{1}, \ldots, f_{r}>$ (finitamente gerado pois $\mathscr{O}_{n}$ é Noetheriano) existe a variedade canonicamente associada $V(I)=\left\{x \in \mathbb{C}^{n} \mid f_{1}(x)=\cdots=f_{r}(x)=0\right\}$.

Como $\mathscr{O}_{n}$ é Noetheriano, todo ideal $Q$ tem decomposição primária, ou seja, $Q=\bigcap_{i=1}^{r} Q_{i}$ onde $Q_{i}$ é um ideal primário. Além disso, os ideais primos $P_{i}=\sqrt{Q_{i}}, i=1, \ldots, r$ são independentes da decomposição, chamados primos associados de $Q$ e denotamos por $\operatorname{Ass}(Q)=\left\{P_{1}, \ldots, P_{n}\right\}$.

Seja $X$ um germe de uma variedade analítica na origem em $\mathbb{C}^{n}, X=X_{1} \cup \cdots \cup X_{r}$ a sua decomposição em componentes irredutíveis. Os $X_{i}$ 's correspondem exatamente aos elementos 
minimais de $\operatorname{Ass}(I(X))$. Assim se $I(X)=\bigcap_{i=1}^{r} Q_{i}$ e $P_{i}=\sqrt{Q_{i}}$ então $X_{i}=V\left(P_{i}\right)$.

Seja $W$ um subconjunto analítico de algum subconjunto aberto $U$ em algum espaço afim e seja $\alpha$ um feixe coerente de ideais em $\mathscr{O}_{U}$.

Em cada ponto $x \in V(\alpha)$ desejamos considerar as componentes do espaço analítico complexo $V(\alpha)$ passando por $x$ que não estão contidas em $|W|$.

Definição 1.24. Consideremos $\alpha_{x}$ o talo de $\alpha$ em $\mathscr{O}_{U, x}$. Seja $S$ o conjunto multiplicativamente fechado $\mathscr{O}_{U, x}-\bigcup p$, onde a união é para todo $p \in A s s\left(\mathscr{O}_{U, x} / \alpha_{x}\right)$ com $|V(p)| \nsubseteq|W|$. Então, definimos $\alpha_{x} / W$ igual a $S^{-1} \alpha_{x} \cap \mathscr{O}_{U, x}$. Assim, $\alpha_{x} / W$ é o ideal em $\mathscr{O}_{U, x}$ consistindo da intersecção destas componentes primárias, $q$, de $\alpha_{x}$ tais que $|V(q)| \nsubseteq|W|$.

Agora, tendo definido $\alpha_{x} / W$ em cada talo, por [27], se executamos essa operação simultaneamente em todos os pontos de $V(\alpha)$, então obtemos um feixe coerente de ideais chamado feixe lacuna; escrevemos esse feixe como $\alpha / W$. Se $V=V(\alpha), V / W$ é denotado pelo espaço analítico complexo $V(\alpha / W)$.

\subsection{Ciclos analíticos}

A referência para detalhes é [7].

Definição 1.25. Seja $X$ um espaço analítico complexo de dimensão $d$. Para $0 \leq k \leq d$, um kciclo em X é uma soma formal finita $\sum_{i=1}^{r} \eta_{i}\left[V_{i}\right]$ onde os $V_{i}$ são subvariedades de $X$ e os $\eta_{i}$ são números inteiros.

Notação: Denotamos por $Z_{k}(X)$ o grupo formado pelos $k$-ciclos em $X$ e $Z(X):=\oplus_{k=0}^{d} Z_{k}(X)$. Os elementos de $Z(X)$ são chamados de ciclos de $X$.

Consideremos agora um espaço analítico complexo $X$ com estrutura reduzida, ou seja, $I(X)=$ $\sqrt{I(X)}=\left\{f \in \mathscr{O}_{n} \mid f^{k} \in I(X)\right.$ para algum inteiro $\left.k>0\right\}$. Sejam $V_{1}, \ldots, V_{r}$ as suas componentes irredutíveis.

Definiçãa 1.26. $O$ ciclo analítico associado a $X$, denotado por $[X]$, é o elemento $\sum_{i=1}^{r} \eta_{i}\left[V_{i}\right]$ de $Z(X)$, onde os $\eta_{V_{i}}$ são definidos como segue: 
Tome um ponto $p$ em $V_{i}$ e considere o germe de $V_{i}$ em $p$. Sejam $\left(V_{i p}\right)_{j}$ as componentes irredutíveis deste germe.

Tome algum dos $\left(V_{i p}\right)_{j}$ e seja $\eta_{V_{i}}$ igual ao comprimento do anel $\left(\mathscr{O}_{V_{i}, p}\right)_{\left(\widetilde{V_{i p}}\right)_{j}}$ onde $\left(\widetilde{V_{i p}}\right)_{j}$ é o ideal primo correspondente $a\left(V_{i p}\right)_{j}$.

Observação 1.27. Massey observa na página 9 de [17] que esse número independe do ponto $p$ e da escolha de $\left(V_{i p}\right)_{j}$.

Com essa notação, é fundamental que, se $f, g \in \mathscr{O}_{U}$ então $[V(f g)]=[V(f)]+[V(g)] \mathrm{e}$, em particular, $\left[V\left(f^{m}\right)\right]=m[V(f)]$.

Sejam $V$ e $W$ dois espaços analíticos complexos irredutíveis em um subconjunto aberto $U$ de algum espaço afim. Dizemos que $V$ e $W$ se interceptam propriamente em $U$ desde que codim $V \cap W=\operatorname{codim} V+\operatorname{codim} W$. Então, neste caso, a intersecção produto de $[V]$ e $[W]$ é definida por $[V] \cdot[W]=[V \cap W]$.

Dois ciclos $\sum m_{i}\left[V_{i}\right]$ e $\sum n_{j}\left[V_{j}\right]$ são ditos que se interceptam propriamente se $V_{i}$ e $W_{j}$ se interceptam propriamente, $\forall i, j$. Então neste caso a intersecção produto é estendida bilinearmente pela definição

$$
\sum m_{i}\left[V_{i}\right] \cdot \sum n_{j}\left[W_{j}\right]=\sum m_{i} n_{j}\left(\left[V_{i}\right] \cdot\left[W_{j}\right]\right)=\sum m_{i} n_{j}\left(\left[V_{i} \cap W_{j}\right]\right)
$$

Se dois ciclos $C_{1}$ e $C_{2}$ se interceptam propriamente e $C_{1} \cdot C_{2}=\sum p_{k}\left[Z_{k}\right]$ onde $Z_{k}$ são irredutíveis então o número de intersecções de $C_{1}$ e $C_{2}$ em $Z_{k},\left(C_{1} \cdot C_{2}\right)_{Z_{k}}$, é $p_{k}$.

Destacamos aqui um resultado clássico de intersecção de ciclos, que será uma ferramenta muito importante para o desenvolvimento do Capítulo 3. A referência para este resultado é [17] (pag. 10) ou [7].

Proposição 1.28. Dados um ponto $p \in U$, uma curva $W$ em $U$ que é irredutível em $p$ e uma hipersuperfície $V(f) \subseteq U$ que intercepta $W$ propriamente em $p$, existe um modo muito útil para calcular o número de intersecções $([W] \cdot[V(f)])_{p}$. Toma-se uma parametrização local $\phi(t)$ de $W$ que leva 0 em $p$, e então $([W] \cdot[V(f)])_{p}=$ mult $_{t} f(\phi(t))=$ o grau do menor termo não nulo. 


\subsection{Fibrado vetorial complexo}

As referências para detalhes são [21] e [28].

Definição 1.29. Um fibrado vetorial complexo é uma terna $\xi=(E, B, \pi)$ onde $E, B$ são espaços topológicos (chamados espaço total e espaço base do fibrado, respectivamente) e $\pi$ : $E \rightarrow B$ é uma aplicação (contínua) sobrejetora, satisfazendo as seguintes condições:

1. para cada ponto $p \in B, \pi^{-1}(p)$ tem uma estrutura de espaço vetorial complexo;

2. para cada ponto $p \in B$ deve existir uma vizinhança $U_{p} \subset B$, um inteiro $n \geq 0$ e um homeomorfismo

$$
h: U_{p} \times \mathbb{C}^{n} \rightarrow \pi^{-1}\left(U_{p}\right)
$$

tal que, para cada $q \in U_{p}$, a correspondência $x \mapsto h(q, x)$ define um isomorfismo entre o espaço vetorial $\mathbb{C}^{n}$ e o espaço vetorial $\pi^{-1}(q)$.

Se pudermos escolher $U$ igual a B, esse fibrado é chamado trivial.

Se todas as fibras de $\xi$ tiverem a mesma dimensão $n$, diremos que $\xi$ tem dimensão (ou posto) $n$. Observe que, devido a $E$ ser localmente trivial , a função $x \mapsto \operatorname{dim} \pi^{-1}(x)$ é localmente constante, logo é constante nas componentes conexas de $B$. Em particular, se $B$ for conexo, $\xi$ terá uma dimensão.

Uma operação importante entre fibrados $\xi_{1}=\left(E_{1}, B_{1}, \pi_{1}\right)$ e $\xi_{2}=\left(E_{2}, B_{2}, \pi_{2}\right)$ é a produto de fibrados, denotada por $\xi_{1} \otimes \xi_{2}$, com base $B_{1} \times B_{2}$ onde a fibra de um ponto $\left(b_{1}, b_{2}\right) \in B_{1} \times B_{2}$ é o produto das fibras de $b_{1} \in B_{1}$ e $b_{2} \in B_{2}$ dos fibrados $\xi_{1}$ e $\xi_{2}$ respectivamente.

Definição 1.30. Sejam $\xi=(E, B, \pi)$ um fibrado, $B^{\prime}$ um espaço topológico e $f: B^{\prime} \rightarrow B$ uma aplicação contínua. O fibrado induzido de $\xi$ por $f$, denotado por $f^{*}$, é o fibrado cujo espaço total $E^{\prime} \subseteq B^{\prime} \times E$ é da forma $\left(b^{\prime}, e\right)$ tal que $f\left(b^{\prime}\right)=\pi(e)$ e a projeção $\pi: E^{\prime} \rightarrow B^{\prime}$ é da forma $\pi^{\prime}\left(b^{\prime}, e\right)=b^{\prime}$.

Exemplo 1.31. $O$ fibrado tangente, denotado por $\tau_{M}$, é o fibrado vetorial com espaço base uma variedade suave $M$, espaço total $T M=\left\{(x, v): x \in M\right.$ e $\left.v \in T_{x} M\right\}$ e projeção canônica.

Definição 1.32. Uma seção de um fibrado vetorial complexo $\xi$ é uma função contínua $s: M \rightarrow$ $E$, a qual leva $p$ dentro da sua fibra correspondente $\pi^{-1}(p)$. 
Exemplo 1.33. Todo fibrado vetorial tem uma seção, chamada seção zero,

$$
\begin{aligned}
s_{0}: M & \rightarrow E \\
p & \mapsto 0 \in E_{p}=\pi^{-1}(p) .
\end{aligned}
$$

Observação 1.34. Se $s: M \rightarrow E$ é uma seção, então $s: M \rightarrow s(M)$ é um homeomorfismo. Assim $s(M)$ é uma variedade.

Definição 1.35. Uma seção do fibrado tangente $\tau_{M}$ é chamada de um campo vetorial em $M$, que denotamos em geral por $v$. Em outras palavras, vé uma aplicação suave que a cada ponto $x \in M$ associa um vetor $v(x) \in T_{x} M$.

Suponha que $M$ tenha dimensão $m$ e seja $U$ uma bola de $\mathbb{R}^{m}$, centrada em um ponto $p$, difeomorfa (via $h$ ) a uma vizinhança em $M$. Suponha também que $v$ seja um campo vetorial em $h^{-1}(U)$, com zero isolado em $h^{-1}(p)$. Temos então que $\tilde{v}=\frac{v \circ h^{-1}(x)}{\left\|v \circ h^{-1}(x)\right\|}$ leva um esfera centrada em $p$ na esfera unitária em $\mathbb{R}^{m}$.

Definição 1.36. $O$ índice do campo $v$ em $p$, denotado por $I(v, p)$, é o grau da aplicação $\tilde{v}$.

Definição 1.37. Um r-campo em um subconjunto $A$ de $M$ é um conjunto $v(r)=v_{1}, \ldots, v_{r}$ de $r$ campos de vetores contínuos definidos em A. Um ponto singular de $v(r)$ é um ponto onde os vetores $\left(v_{i}\right)$ não são linearmente independentes. Um r-campo não singular é chamado de um r-frame. Quando $r=1$, dizemos simplesmente frame.

Para esta definição mantivemos a denominação do conceito em inglês, frame, por não encontrar designação para este termo em português.

\subsection{Variedades polares - definidas por Lê e Teissier}

Nesta seção apresentamos o conceito de Variedades Polares, introduzido por Lê e Teissier em [29] e [13], que é uma ferramenta de grande importância em vários domínios da matemática, como Teoria de Singularidades, Teoria de Estratificação, Geometria Algébrica e Classes Características, e é amplamente utilizado neste trabalho.

Definição 1.38. Seja $V \subset \mathbb{C}^{N}$ uma subvariedade algébrica (ou analítica), reduzida e equidimensional (dimensão d) com $V_{\text {reg }}$ denotando a parte regular de $V$. Para $0 \leq k \leq d$, dado $D_{d-k+1}$ 
um subespaço vetorial complexo de codimensão $d-k+1$, definimos a $k$-ésima variedade polar de Lê-Teissier de V com relação a $D_{d-k+1}$ como sendo

$$
P_{k}\left(V, D_{d-k+1}\right)=\overline{\left\{p \in V_{\text {reg }} \mid \operatorname{dim}\left(T_{p} V_{\text {reg }} \cap D_{d-k+1}\right) \geq k\right\}},
$$

onde dim denota a dimensão complexa.

Como estamos no caso complexo, é possível provar que existe um aberto denso U na grassmaniana de $(N-d+k-1)$-planos de $\mathbb{C}^{N}$, denotada por $G(N-d+k-1, N)$, tal que para todo $D_{d-k+1} \in U$ a codimensão de $P_{k}\left(V, D_{d-k+1}\right)$ é igual a $k$.

Assim, para $0 \leq k \leq d$, definimos a $k$-ésima variedade polar genérica de Lê-Teissier de $V$ (ou simplesmente a k-ésima variedade polar de Lê-Teissier de $V$ ), que denotamos por $P_{k}(V)$, se $P_{k}(V)=P_{k}\left(V, D_{d-k+1}\right)$ para algum $D_{d-k+1} \in U$.

\subsubsection{Multiplicidades polares}

Inicialmente vamos definir o conceito de multiplicidade de um ponto em uma subvariedade. Existe uma maneira algébrica de obter esta multiplicidade (Ver [30]), que é chamada multiplicidade de Hilbert-Samuel.

Definição 1.39. Sejam $V$ uma subvariedade de dimensão $k$ contida em uma variedade regular $M$ e p um ponto de V. Seja D um pequeno polidisco centrado em p. Consideremos a projeção $P$ sobre um plano genérico de dimensão $k$. Seja q um ponto de $P(D)-P(p)$ e $\delta(p)$ um pequeno polidisco centrado em $q$. A multiplicidade de $V$ em $p$, denotada por $m_{p} V$, é o número de folhas de $P^{-1}(\delta(p)) \cap D \cap V$.

Sejam $V$ uma subvariedade analítica complexa reduzida e equidimensional (dimensão $d$ ) e $P_{k}(V)$ a variedade polar de Lê-Teissier de $V \operatorname{com} k=0, \cdots, d$.

Definição 1.40. Dado $p \in P_{k}(V)$ definimos a sequência de multiplicidades (polares) de LêTeissier em p como sendo a sequência $\left(m_{p} P_{0}(V), m_{p} P_{1}(V), \cdots, m_{p} P_{d}(V)\right)$.

\subsection{Estratificação de Whitney}

No estudo de singularidades de conjuntos, a principal ideia é utilizar a teoria de estratificação. Intuitivamente, podemos pensar numa estratificação como uma decomposição de um espaço 
singular em variedades regulares, chamados estratos, com algum tipo de controle de como estes estratos se encontram. Nesta seção vamos definir um tipo especial de estratificação chamada estratificação de Whitney, onde as referências utilizadas são [31] e [11].

Definição 1.41. Sejam $M$ uma variedade suave e $V$ uma variedade analítica de $M$. Uma estratificação de $V$ é uma partição de $V$ localmente finita em subvariedades $\left\{V_{\alpha}\right\}$ de $M$, chamadas de estratos.

\section{Condição de fronteira}

Dizemos que a estratificação $\left\{V_{\alpha}\right\}$ de $V$ satisfaz a condição de fronteira, se para dois estratos $V_{\alpha}$ e $V_{\beta}$, tais que $V_{\alpha} \cap \bar{V}_{\beta} \neq \emptyset$, então $V_{\alpha} \subset \bar{V}_{\beta}$.

\section{Condições de Whitney}

Dizemos que a estratificação $\mathscr{B}=\left\{V_{\alpha}\right\}$ de $V$ satisfaz as condições de Whitney, se para todo $\operatorname{par}\left(V_{\alpha}, V_{\beta}\right)$ de estratos tais que $V_{\beta}$ esteja no fecho de $V_{\alpha}$ e para todo ponto $y$ de $V_{\beta}$ temos:

(a) Para toda sequência de pontos $x_{i}$ de $V_{\alpha}$ convergindo para $y$, tal que as direções dos espaços tangentes $T_{x_{i}} V_{\alpha}$ convergem para um hiperplano $T$, então $T$ contém $T_{y} V_{\beta}$.

(b) Se além disso temos uma sequência $y_{i}$ de pontos de $V_{\beta}$ convergindo para $y$, tal que as direções das secantes $\overline{x_{i} y_{i}}$ convergem (no espaço projetivo) para $l$, então $T$ contém $l$.

Estas são as chamadas condição $(a)$ e condição $(b)$ de Whitney.

Observação 1.42. Whitney mostrou em [31] que toda variedade analítica complexa admite uma estratificação satisfazendo estas duas condições.

Definição 1.43. Uma estratificação satisfazendo a condição de fronteira e as condições $(a) e$ (b) de Whitney é chamada de estratificação de Whitney.

Uma importante propriedade envolvendo a estratificação de Whitney é que ela é preservada por transversalidade como segue.

Proposição 1.44. Sejam $\mathscr{B}=\left\{V_{\alpha}\right\}$ uma estratificação de $V \subset M$ e $f: N \rightarrow M$ uma aplicação suave entre variedades suaves, que é transversal a todos os estratos $V_{\alpha}$, ou seja,

$$
d_{x} f\left(T_{x} N\right)+T_{f(x)} V_{\alpha}=T_{f(x)} M \text { para todo } x \in f^{-1}\left(V_{\alpha}\right) .
$$

Se $\mathscr{B}=\left\{V_{\alpha}\right\}$ é uma estratificação de Whitney de $V$ então $\mathscr{B}^{\prime}=\left\{f^{-1}\left(V_{\alpha}\right)\right\}$ é uma estratificação de Whitney de $f^{-1}(V)$. 


\subsubsection{Estratificação de Whitney minimal - descrita por Lê e Teissier}

Seja $V$ uma variedade analítica (afim) reduzida e equidimensional (dimensão $d$ ). A estratificação de Whitney minimal de $V$ é uma estratificação de Whitney consequente do trabalho de Lê e Teissier ([13]) e que foi definida em termos das sequências de multiplicidades (polares) de Lê-Teissier.

\section{Construção da Estratificação de Whitney minimal}

De acordo com Lê e Teissier em [13], podemos obter uma sequência de subvariedades algébricas de $V, F_{0} \supset F_{1} \supset \cdots \supset F_{k} \cdots$, da seguinte maneira:

Seja $F_{0}=V$ e $F_{1}=\Sigma(V)$, o conjunto singular de $V$. Denotemos por $\left(F_{1, j_{1}}\right)_{j_{1} \in J_{1}}$ as componentes de $F_{1}$ e definimos $F_{2}$ como sendo a união do conjunto singular de $F_{1}$ com o conjunto dos pontos $p$ em $F_{1, j_{1}}$ tal que a sequência $\left(m_{p} P_{0}(V), \cdots, m_{p} P_{d}(V)\right)$ de multiplicidades polares de Lê-Teissier é diferente de uma calculada em um ponto genérico de $F_{1, j_{1}}$.

Denotemos por $\left(F_{2, j_{2}}\right)_{j_{2} \in J_{2}}$ as componentes de $F_{2}$. Em geral, seja $F_{k}$ a união do conjunto singular de $F_{k-1}$ com o conjunto dos pontos em $F_{k-1, j_{k-1}}$ tal que a sequência de multiplicidades polares de Lê-Teissier é diferente de uma calculada em um ponto genérico de $F_{k-1, j_{k-1}}$.

Proposição 1.45. (Ver [13]-Corolário 6.1.7) $\mathscr{B}=\left\{F_{i, j} \backslash \bigcup_{k>i} F_{k, l}\right\}$ é uma estratificação de Whitney de $V$ e é chamada estratificação canônica ou minimal.

\subsection{Homologia e cohomologia}

Um complexo de cadeias $C=\left(C_{q}, \partial_{q}\right)$ é definido por um par de sequências de grupos abelianos $C_{q}$ e homomorfismos $\partial_{q}: C_{q} \rightarrow C_{q-1}$ tal que $\partial_{q-1} \circ \partial_{q}=0$ para todo $q \in \mathbb{Z}$. Os elementos de $C_{q}$ são chamados de $q$-cadeias e os homomorfismos $\partial_{q}$ de operadores bordo. Consideremos os seguintes subgrupos dos grupos de cadeias $C_{q}: Z_{q}(C)=\operatorname{Ker}\left(\partial_{q}\right)$ chamado grupo dos $q$-ciclos e $B_{q}(C)=\operatorname{Im}\left(\partial_{q-1}\right)$ chamado grupo dos $q$-bordos. São ambos subgrupos normais de $C_{q}$ e, como $\partial_{q-1} \circ \partial_{q}=0, B_{q}(C) \subset Z_{q}(C)$.

Definição 1.46. O grupo quociente $H_{q}(C):=\frac{Z_{q}(C)}{B_{q}(C)}$ é chamado grupo de homologia $q$-dimensional do complexo $C$. 
Definimos o grupo de cocadeias por $C^{q}(G):=\operatorname{Hom}\left(C_{q}, G\right)=\left\{f: C_{q} \rightarrow G\right.$ homomorfismo $\}$, onde $G$ é um grupo abeliano fixado. Por sua vez, o operador cobordo é definido como o dual do operador bordo e denotado por $\delta^{q}: C^{q-1}(G) \rightarrow C^{q}(G)$.

A partir disso, definimos o grupo dos $q$-cociclos como $Z^{q}(C, G)=\operatorname{Ker}\left(\delta^{q-1}\right)$ e o grupo dos $q$-cobordos como $B^{q}(C, G)=\operatorname{Im}\left(\delta_{q}\right)$. Como também temos $B^{q}(C, G) \subset Z^{q}(C, G)$ temos a seguinte definição.

Definição 1.47. $O$ q-ésimo grupo de cohomologia de $C$ é definido por $H^{q}(C, G):=\frac{Z^{q}(C, G)}{B^{q}(C, G)}$.

Notação: Para $c^{q} \in C^{q}(G)$ e $c_{q} \in C_{q}$, denotamos $\left\langle c^{q}, c_{q}>:=c^{q}\left(c_{q}\right) \in G\right.$.

Existem várias teorias de homologia (cohomologia). Nesta seção será feita uma breve apresentação sobre a Teoria de Homologia (Cohomologia) Simplicial, para fixar notações e especificar algumas nomenclaturas utilizadas durante o trabalho.

\subsubsection{Homologia (cohomologia) simplicial}

Nesta teoria são definidos grupos de homologia e cohomologia de um poliedro e, mais geralmente, de um espaço topológico triangulável, isto é, homeomorfo a um poliedro.

Trata-se de uma situação abrangente já que toda variedade suave é triangulável (Vide [22]).

A referência para detalhes é [15].

Definição 1.48. Um simplexo $s$ de dimensão n é o menor subconjunto convexo de $\mathbb{R}^{m}$ que contém os pontos $a_{0}, \cdots, a_{n} \in \mathbb{R}^{m}$ (vértices), onde $a_{1}-a_{0}, \cdots, a_{n}-a_{0}$ são linearmente independentes.

Notação: $s=<a_{0}, \cdots, a_{n}>$.

Uma face de um simplexo é qualquer simplexo que tenha por vértices um subconjunto do conjunto de vértices de s.

Definição 1.49. Um poliedro é um subconjunto $K \subset \mathbb{R}^{m}$, no qual foi especificada uma coleção finita de simplexo de $\mathbb{R}^{m}$, chamados simplexos de $K$, de modo que as condições abaixo são satisfeitas:

1) Todo ponto de $K$ pertence a algum simplexo de $K$ (ou seja, $K$ é a reunião dos seus simplexos);

2) Toda face de um simplexo de $K$ é ainda um simplexo de $K$; 
3) Se s e t são simplexos de K então $s \cap t$ é vazio ou é uma face comum a s e t (e portanto é um simplexo de $K)$.

Definição 1.50. A dimensão de um poliedro é a maior dimensão de um de seus simplexos.

Definição 1.51. Um subpoliedro do poliedro $K$ é um poliedro L cujos simplexos são também simplexos de $K$.

Definição 1.52. $O$ esqueleto $r$-dimensional de um poliedro $K$ é o subpoliedro $K^{r}$ formado pelos simplexos de $K$ que têm dimensão $\leq r$.

Definição 1.53. Uma triangulação de um espaço topológico $X$ é um par $(K, h)$ onde $K$ é um poliedro e $h: K \rightarrow X$ é um homeomorfismo.

Existem $(r+1)$ ! maneiras de ordenar os vértices de um simplexo de dimensão $r$. Consideremos equivalentes duas destas ordenações quando uma delas puder ser obtida da outra por meio de uma permutação par dos $r+1$ vértices. Há duas classes de equivalência segundo esta relação. Cada uma dessas classes chama-se uma orientação do simplexo. Escrevemos $s=$ $\left[a_{0}, a_{1}, \cdots, a_{r}\right]$ para indicar o simplexo $s=<a_{0}, a_{1}, \cdots, a_{r}>$ munido da orientação determinada pela ordem $a_{0}<a_{1}<\cdots<a_{r}$

Denotemos por $\triangle_{n}:=\left[e_{0}, \cdots, e_{n}\right], e_{0}=(0, \cdots, 0), e_{i}=(0, \cdots, 0, \underbrace{1}_{i}, 0, \cdots, 0) \in \mathbb{R}^{m}$, com $i=1, \cdots, n$ e $S_{n}(X):=\left\{\sigma \mid \sigma: \triangle_{n} \rightarrow X\right.$ contínua $\}$.

Dado $A$ um anel comutativo com unidade, definimos $C_{n}(X, A)$ o grupo abeliano livre gerado por $S_{n}(X)$ com coeficientes em $A$.

O operador bordo $\partial_{n}: C_{n}(X, A) \rightarrow C_{n-1}(X, A)$ é definido por $\partial_{n} \sigma=\sum_{i=0}^{r} \sigma_{(i)}$, onde

$$
\sigma_{(i)}\left(v_{1}, \cdots, v_{n}\right)=\left(v_{1}, \cdots, \hat{v}_{i}, \cdots, v_{n}\right) \text {. }
$$

Definição 1.54. Definimos os grupos de homologia simplicial de $X, H_{i}(X, A)$, como sendo os quocientes $\frac{\operatorname{Ker} \partial_{i}}{\operatorname{Im} \partial_{i-1}}$.

Como mostramos no início desta seção, podemos definir o grupo de cohomologia de $X$, $H^{i}(X, A)$, a partir do seu grupo de homologia $H_{i}(X, A)$.

Uma operação importante envolvendo os grupos de homologia e cohomologia, utilizada neste trabalho é a chamada produto cap. 
Definição 1.55. A operação $\cap$ é uma aplicação bilinear dada por:

$$
\sigma \cap \psi=\left.\psi\left(\sigma_{\left[a_{0}, \cdots, a_{q}\right]}\right)\right|_{\left[a_{q}, \cdots, a_{p}\right]},
$$

onde $\sigma: \triangle_{p} \rightarrow X \operatorname{com} \triangle_{p}=\left[e_{0}, \cdots, e_{p}\right], e_{i}=(0, \cdots, 0, \underbrace{1}_{i}, 0, \cdots, 0) e \psi \in C^{q}(X, A)$

$O$ produto cap induz um produto nas respectivas classes de homologia e cohomologia,

$$
\cap: H_{p}(X, A) \times H^{q}(X, A) \rightarrow H_{p-q}(X, A)
$$

Notação: Escrevemos simplesmente $H_{p}(X)$ quando $A=\mathbb{Z}$.

Seja $Y$ um subconjunto de $X$. Podemos definir $C_{q}(X, Y, A)$ como sendo o grupo quociente do grupo $C_{q}(X, A)$ pelo grupo $C_{q}(Y, A)$ e de maneira análoga podemos definir os grupos de homologia e cohomologia.

\subsection{Fibração de Milnor}

O Teorema da Fibração de Milnor é um dos resultados centrais no estudo de variedades analíticas complexas singulares. Este resultado define uma fibração de $S_{\varepsilon}-K$ sobre $S^{1}$ com fibra $F_{\theta}$. A referência para detalhes é [19].

Teorema 1.56. Se $z^{o}$ é um ponto qualquer de $V$ e se $S_{\varepsilon}$ é uma esfera suficientemente pequena centrada em $z^{o}$ então $S_{\varepsilon}-K$ é um fibrado diferenciável localmente trivial sobre $S^{1}$, com projeção $\phi(z)=\frac{f(z)}{|f(z)|}$ e fibra $F_{\theta}=\phi^{-1}\left(e^{i \theta}\right)$.

Teorema 1.57. Se o número complexo $c \neq 0$ tem módulo suficientemente pequeno, então a hipersuperfície complexa $f^{-1}(c)$ intercepta o disco $\stackrel{\circ}{D}_{\varepsilon}$ numa variedade diferenciável difeomorfa a fibra $F_{\theta}$.

O teorema anterior nos leva a considerar uma fibração equivalente, porém mais conveniente, que vive dentro da bola de raio $\varepsilon$. Durante este trabalho, esta fibração de Milnor é usada como descrita abaixo.

Para todo $\varepsilon>0$, seja $\stackrel{\circ}{B}_{\varepsilon}$ a bola aberta de raio $\varepsilon$ centrada na origem em $\mathbb{C}^{n+1}$. Para todo $\eta>0$, sejam $\mathbb{D}_{\eta}$ o disco fechado centrado na origem em $\mathbb{C}$ e $\partial \mathbb{D}_{\eta}$ denotando sua fronteira, que é um círculo de raio $\eta$. Então, tendo fixado uma função analítica, $f$, existe $\varepsilon_{0}>0$ tal que, para todo $\varepsilon$ tal que $0<\varepsilon \leq \varepsilon_{0}$, exista $\eta_{\varepsilon}>0$ tal que, para todo $\eta$ tal que $0<\eta \leq \eta_{\varepsilon}$, a restrição de $f$ 
por uma aplicação $\stackrel{\circ}{B}_{\mathcal{\varepsilon}} \cap f^{-1}\left(\partial \mathbb{D}_{\eta}\right) \rightarrow \partial \mathbb{D}_{\eta}$ é uma fibração localmente trivial suave cujo tipo de difeomorfismo independe da escolha de $\varepsilon$ e $\eta$.

Essa fibração é chamada de Fibração de Milnor de $f$ na origem e a fibra é a fibra de Milnor de $f$ na origem, que a partir daqui é denotada por $F_{f, 0}$. Quando $f$ tem singularidade isolada em 0 , o teorema abaixo caracteriza o tipo de homotopia da fibra $F_{f, 0}$.

Teorema 1.58. Cada fibra $F_{f, 0}$ tem o mesmo tipo de homotopia de um bouquet $S^{n} \vee \ldots \vee S^{n}$ de esferas.

Definição 1.59. O número de esferas $S^{n}$ do bouquet, ou o número de geradores da homologia média da fibra $F_{f, 0}$ é chamado número de Milnor de $f$ em 0 e é denotado por $\mu(f)$.

No caso de polinômios quase homogêneos, Milnor e Orlik em [18] mostram como calcular o número de Milnor.

Teorema 1.60. Seja $f\left(z_{0}, \ldots, z_{n}\right)$ um polinômio quase-homogêneo do tipo $\left(r_{0}, \ldots, r_{n} ; d\right)$ com um ponto crítico isolado na origem. Então

$$
\mu=\frac{\left(d-r_{0}\right)\left(d-r_{1}\right) \cdots\left(d-r_{n}\right)}{r_{0} \ldots r_{n}} .
$$




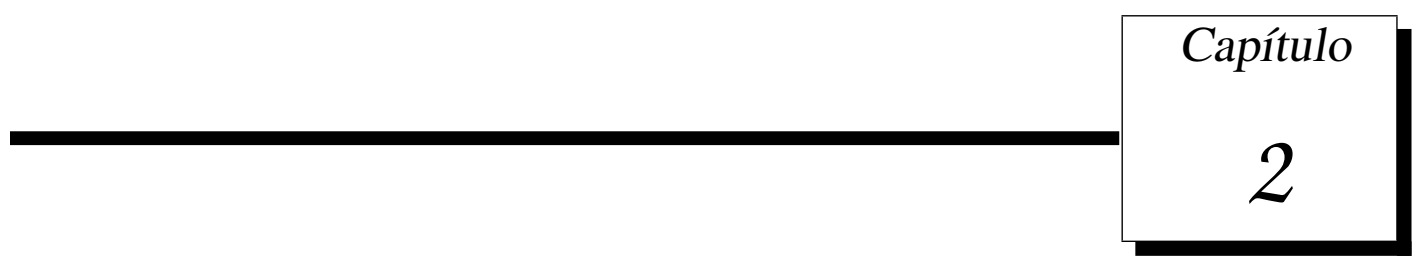

\section{Números de Lê}

Neste capítulo vamos definir os números de Lê para germes de funções holomorfas como definido por Massey em [17] e descrever brevemente alguns resultados utilizados neste trabalho. Em seguida, definimos de maneira natural os números de Lê associados a uma hipersuperfície e descrevemos resultados análogos associados a esta.

\subsection{Números de Lê de germes de funções holomorfas}

A referência para detalhes é [17].

Denotemos por $U$ um subconjunto aberto de $\mathbb{C}^{n+1}$ contendo a origem, $h:(U, 0) \rightarrow(\mathbb{C}, 0)$ um germe de uma função holomorfa tal que $\operatorname{dim}_{0} \Sigma(h)=s$ e $z=\left(z_{0}, \cdots, z_{n}\right)$ um sistema linear de coordenadas em $\mathbb{C}^{n+1}$.

Definição 2.1. Para $0 \leq k \leq n$, a $k$-ésima variedade polar (relativa), $\Gamma_{h, z}^{k}$, de $h$ com respeito a $z$ é o espaço analítico complexo $V\left(\frac{\partial h}{\partial z_{k}}, \ldots, \frac{\partial h}{\partial z_{n}}\right) / \Sigma(h)$. Se a escolha do sistema de coordenadas é clara, escrevemos simplesmente $\Gamma_{h}^{k}$.

Assim, ao nível de ideais, $\Gamma_{h, z}^{k}$ consiste das componentes de $V\left(\frac{\partial h}{\partial z_{k}}, \ldots, \frac{\partial h}{\partial z_{n}}\right)$ que não estão contidas em $|\Sigma(h)|$. Note, em particular, que $\Gamma_{h, z}^{0}$ é vazio. Naturalmente, definimos o $k$-ésimo ciclo polar de $h$ com respeito a $z$ sendo o ciclo analítico $\left[\Gamma_{h, z}^{k}\right]$.

Definição 2.2. Para $0 \leq k \leq n$, definimos o $k$-ésimo ciclo de Lê de h com respeito a $z$, denotado $\operatorname{por}\left[\Lambda_{h, z}^{k}\right]$, como sendo

$$
\left[\Gamma_{h, z}^{k+1} \cap V\left(\frac{\partial h}{\partial z_{k}}\right)\right]-\left[\Gamma_{h, z}^{k}\right] .
$$


Desta definição podemos comcluir que todas as componentes deste ciclo estão contidas no conjunto crítico de $h$.

Como os ciclos de Lê já são dados como ciclos analíticos, omitiremos os colchetes e escreveremos simplesmente $\Lambda_{h, z}^{k}$ para denotar o ciclo de Lê.

Definição 2.3. Definimos o $k$-ésimo número de Lê de $h$ em $p$ com respeito $a z, \lambda_{h, z}^{k}(p)$, como sendo igual ao número de intersecções

$$
\left(\Lambda_{h, z}^{k} \cdot V\left(z_{0}-p_{0}, \ldots, z_{k-1}-p_{k-1}\right)\right)_{p}
$$

desde que essa intersecção seja 0-dimensional em p.

Se essa intersecção não é puramente 0-dimensional em p, então dizemos que o k-ésimo número de Lê (de h em p com respeito a z) está indefinido.

Aqui, quando $k=0$, queremos dizer que

$$
\lambda_{h, z}^{0}(p)=\left(\Lambda_{h, z}^{0} \cdot U\right)_{p}=\left(\left[\Gamma_{h, z}^{1} \cap V\left(\frac{\partial h}{\partial z_{0}}\right)\right]\right)_{p}=\left(\left[\Gamma_{h, z}^{1}\right] \cdot\left[V\left(\frac{\partial h}{\partial z_{0}}\right)\right]\right)_{p}
$$

Exemplo 2.4. Seja $h=y^{2}-x^{3}-t x^{2}$; esse é o guarda-chuva de Whitney. Fixamos o sistema de coordenadas $z=(t, x, y)$.

Temos $\Sigma(h)=V\left(-x^{2},-3 x^{2}-2 t x, 2 y\right)=V(x, y)$. Assim o conjunto crítico de $h$ é 1-dimensional, enquanto a dimensão de toda componente de $V\left(\frac{\partial h}{\partial y}\right)$ é 2 .

Daí, $V\left(\frac{\partial h}{\partial y}\right)$ não possui nenhuma componente contida em $\Sigma(h)$ e, por isso, começamos calculando variedades polares com $\Gamma_{h}^{2}$.

$$
\begin{gathered}
\text { Temos } \Gamma_{h}^{2}=V\left(\frac{\partial h}{\partial y}\right)=V(2 y)=V(y) \text { e, portanto, } \\
\Gamma_{h}^{2} \cap V\left(\frac{\partial h}{\partial x}\right)=V(y) \cap V\left(-3 x^{2}-2 t x\right)=V\left(y,-3 x^{2}-2 t x\right) . \\
\Gamma_{h}^{1}=\left(\Gamma_{h}^{2} \cap V\left(\frac{\partial h}{\partial x}\right)\right) / \Sigma(h)=V\left(y,-3 x^{2}-2 t x\right) / V(x, y)=V(y,-3 x-2 t) .
\end{gathered}
$$

Da definição do ciclo de Lê obtemos 


$$
\begin{aligned}
\Lambda_{h}^{1} & =\left[V\left(y,-3 x^{2}-2 t x\right)\right]-[V(y,-3 x-2 t)] \\
& =([V(y, x)]+[V(y,-3 x-2 t)])-[V(y,-3 x-2 t)] \\
& =[V(y, x)] .
\end{aligned}
$$

Assim, $\Lambda_{h}^{1}$ tem como seu conjunto básico o eixo $t$ e esse eixo ocorre com multiplicidade 1. Agora achamos $\Lambda_{h}^{0}=\left[\Gamma_{h}^{1} \cap V\left(\frac{\partial h}{\partial t}\right)\right]=\left[V(y,-3 x-2 t) \cap V\left(-x^{2}\right)\right]=2[V(t, x, y)]=2[0]$.

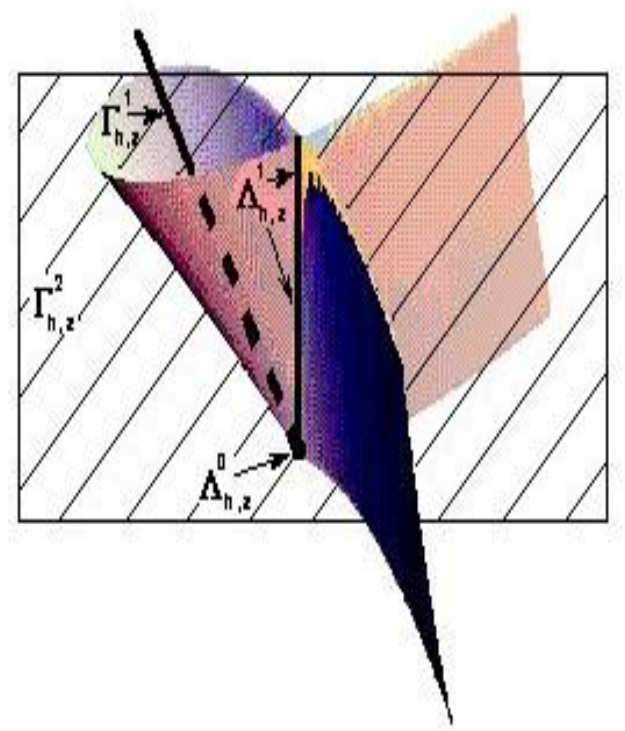

Finalmente calculamos os números de Lê: $\lambda_{h}^{1}(0)=(V(y, x) \cdot V(t))_{0}=1$ e $\lambda_{h}^{0}(0)=2$.

Dentre as várias propriedades que envolvem os ciclos e os números de Lê, destacamos duas que serão diretamente necessárias ao desenvolvimento deste trabalho. A primeira descreve como calcular indutivamente os ciclos de Lê e a segunda mostra como os números de Lê se comportam tomando seções hiperplanas.

Proposição 2.5. (Ver [17], 1.18) Se, para todo j com $0 \leq j \leq k, \Lambda_{h, z}^{j}$ é puramente j-dimensional em p, então os ciclos $\left[\Gamma_{h, z}^{k+1} \cap V\left(\frac{\partial h}{\partial z_{k}}\right)\right] e\left[\Gamma_{h, z}^{k+1}\right] \cdot\left[V\left(\frac{\partial h}{\partial z_{k}}\right)\right]$ são iguais em p. Consequentemente, se sé a dimensão do conjunto crítico de h temos que

$$
\left[\Gamma_{h, z}^{s+1}\right]=\left[V\left(\frac{\partial h}{\partial z_{s+1}}, \ldots, \frac{\partial h}{\partial z_{n}}\right)\right] e\left[\Gamma_{h, z}^{i+1}\right] \cdot\left[V\left(\frac{\partial h}{\partial z_{i}}\right)\right]=\left[\Gamma_{h, z}^{i}\right]+\left[\Lambda_{h, z}^{i}\right], 0 \leq i \leq s .
$$


Proposição 2.6. (Ver [17], 1.21) Consideremos $p \in \Sigma(h)$. Suponhamos que $\Sigma(h) \cap V\left(z_{0}-p_{0}\right)=$ $\Sigma\left(h_{\left.\left.\right|_{V\left(z_{0}-p_{0}\right)}\right)}\right)$ e usemos as coordenadas $\tilde{z}=\left(z_{1}, \ldots, z_{n}\right)$ para $V\left(z_{0}-p_{0}\right)$. Se $\lambda_{h, z}^{i}(p)$ estiver definido para todo i e $p \notin \Gamma_{h, z}^{1}$ então,

$$
\lambda_{h_{\mid V\left(z_{0}-p_{0}\right)}^{i}, \tilde{z}}^{i}(p)=\lambda_{h, z}^{i+1}(p)
$$

\subsubsection{Coordenadas pré-polares}

Com as definições apresentadas não é possível garantir a existência dos números de Lê, que depende da escolha do sistema de coordenadas. Contudo Massey exibe em [17] (Teorema 1.28) um sistema de coordenadas que é genérico com respeito a um certo tipo de estratificação da hipersuperfície $V(h)$ no qual os números de Lê estão sempre definidos.

Definição 2.7. Uma boa estratificação de h no ponto $p \in V(h)$ é uma estratificação analítica, $\mathscr{B}$, da hipersuperfície $V(h)$ em uma vizinhança $U$ de $p$ tal que a parte suave de $V(h)$ é um estrato e de modo que a estratificação satisfaça $a_{h}$ condição de Thom com respeito a $U-V(h)$, ou seja, se $q_{i}$ é uma sequência de pontos em $U-V(h)$ tal que $q_{i} \rightarrow q \in S \in \mathscr{B}$ e $T_{q_{i}} V\left(h-h\left(q_{i}\right)\right)$ converge para algum hiperplano $T$, então $T_{q} S \subseteq T$.

Definição 2.8. Sejam $z=\left(z_{0}, \cdots, z_{n}\right)$ uma escolha linear de coordenadas para $\mathbb{C}^{n+1}$ e $p \in V(h)$ onde $s=\operatorname{dim}_{p} \Sigma h$. Dizemos que $z=\left(z_{0}, \cdots, z_{n}\right)$ é pré-polar para $h$ em $p$ se existe uma boa estratificação de h no ponto p tal que $V\left(z_{0}-p_{0}\right)$ intercepta transversalmente todos os estratos $S_{\alpha}$ - exceto o possível estrato $\{p\}$ - e para todo $j$ tal que $1 \leq j \leq s-1 V\left(z_{j}-p_{j}\right)$ intercepta transversalmente todos os $S_{\alpha} \cap V\left(z_{0}-p_{0}, \cdots, z_{j-1}-p_{j-1}\right)$ - exceto também o possível estrato $\{p\}$.

Teorema 2.9. (Ver $[17], 1.28)$ Sejam $p \in V(h)$ com $s=\operatorname{dim}_{p} \Sigma h$ e $z=\left(z_{0}, \cdots, z_{n}\right)$ um sistema pré-polar para h em $p$. Então, para todo $i$ com $1 \leq i \leq s, \lambda_{h, z}^{i}(p)$ está definido. Além disso, $\Gamma_{h, z}^{i} \cap V\left(z_{0}-p_{0}, \cdots, z_{i-1}-p_{i-1}\right)$ é vazio ou 0 -dimensional em $p$ com $1 \leq i \leq s$.

Observação 2.10. Outra coisa a ser ressaltada é que, mesmo sendo o sistema de coordenadas pré-polares uma escolha genérica, dois sistemas deste tipo podem determinar números de Lê distintos. Porém, quando variamos a escolha linear de coordenadas z para as quais os números de Lê estão definidos em um certo ponto p, é possivel achar um valor genérico para cada número de Lê $\lambda_{h, z}^{i}(p)$. Por [17] Teorema 10.19 sabemos que tais valores genéricos existem e que 
são invariantes analíticos. Estes números são chamados números de Lê genéricos de hem p e são denotados simplesmente por $\lambda_{h}^{i}(p)$.

Além disso, os números de Lê genéricos são constantes ao longo de cada estrato de qualquer estratificação de Whitney de $V(h)$, também por [17] Teorema 10.19.

\subsection{Números de Lê de hipersuperfícies}

Seja $Z$ uma hipersuperfície contida em uma variedade suave complexa compacta $X$ de dimensão $n+1$. Como toda variedade analítica é fechada e $X$ é compacta, temos que $Z$ é compacta. Assim existem uma cobertura aberta e finita $\left\{U_{i}\right\}_{i=1}^{r}$ de $Z$ em $X$ e funções holomorfas $f_{i}: U_{i} \rightarrow \mathbb{C}$ tais que $U_{i} \cap Z=f_{i}^{-1}(0)$ e $f_{\left.i\right|_{U_{i} \cap U_{j}}}=f_{j_{U_{i} \cap U_{j}}}, \forall i, j$.

Por definição de variedade, podemos supor que esses abertos são tais que para cada um deles existe uma função biholomorfa $h_{i}: U_{i} \rightarrow h\left(U_{i}\right)=V_{i}$, onde $V_{i}$ é um aberto de $\mathbb{C}^{n+1}$.

Definição 2.11. Seja $p \in Z$. Definimos o k-ésimo número de Lê de Z em p como sendo

$$
\lambda^{k}(p):=\lambda_{g_{i}}^{k}(q)
$$

onde $p \in U_{i}, q=h_{i}(p), g_{i}=f_{i} \circ h_{i}^{-1}$ e $\lambda_{g_{i}}^{k}(q)$ é o k-ésimo número de Lê genérico do germe da função holomorfa $g_{i}$.

Este número está bem definido pois se escolhemos outro aberto $U_{j}$ contendo $p, f_{i}$ e $f_{j}$ coincidem na intersecção e esse é um conceito local.

Lema 2.12. Os números de Lê de Z são constantes em cada estrato (todos conexos) da estratificação de Whitney $\mathscr{B}=\left\{S_{\alpha}\right\}$ de $Z$.

Demonstração. Como Sing $Z=\bigcup_{i=1}^{r} \operatorname{Sing} f_{i}^{-1}(0)$, então $\left\{S_{\alpha} \cap U_{j}\right\}_{\alpha}$ é uma estratificação de Whitney de $f_{j}^{-1}(0)$, para todo $j \in\{1, \cdots, r\}$. Denote por $s$ a dimensão de Sing $Z$.

Um ponto $p$ é singular de $f_{j}$ se $h_{j}(p)$ é singular de $g_{j}$ e, consequentemente $\left\{h_{j}\left(S_{\alpha} \cap U_{j}\right)\right\}_{\alpha}$ é uma estratificação de Whitney de $g_{j}^{-1}(0)$.

Pelo Teorema 10.19 de [17] os números de Lê genéricos são constantes ao longo de cada estrato de uma estratificação de Whitney de $g_{j}^{-1}(0)$, então eles são constantes em $S_{\alpha} \cap U_{j}$, para cada $\alpha$. Assim. denote por $\lambda_{S_{\alpha}, j}^{i}$ o i-ésimo número de Lê em $S_{\alpha} \cap U_{j}$. 
Temos que $S_{\alpha}=\bigcup_{k=1}^{r}\left(S_{\alpha} \cap U_{k}\right)$. Como $S_{\alpha}$ é conexo então $U_{k} \cap U_{j} \neq \emptyset$ para todo $k, j$ tal que $S_{\alpha} \cap U_{k} \neq \emptyset$ e $S_{\alpha} \cap U_{j} \neq \emptyset$.

Como $f_{k} \equiv f_{j}$ em $U_{k} \cap U_{j} \Rightarrow \lambda_{S_{\alpha}, k}^{i}=\lambda_{S_{\alpha}, j^{\prime}}^{i}$. Assim, denotemos por $\lambda_{S_{\alpha}}^{i}=\lambda_{S_{\alpha, k}}^{i}$ o i-ésimo número de Lê em $S_{\alpha}$. 


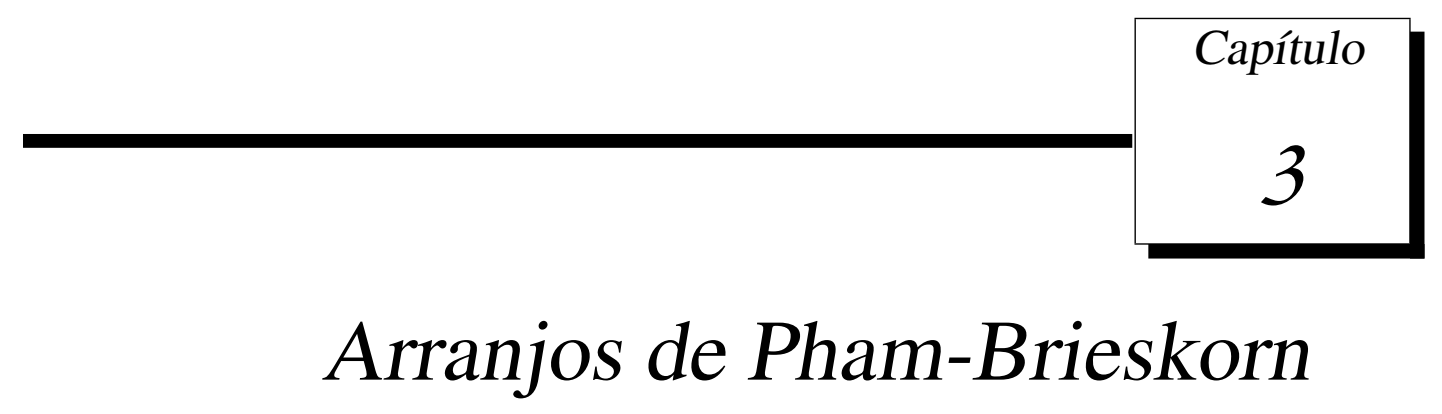

Neste capítulo apresentamos uma descrição dos números de Lê na origem de polinômios que são produtos de polinômios de Pham-Brieskorn do mesmo tipo, os quais denominamos de arranjos de Pham-Brieskorn.

Definição 3.1. Dizemos que $f \in \mathbb{C}\left[x_{0}, \ldots, x_{n}\right]$ é um polinômio de Pham-Brieskorn se

$$
f=x_{0}^{a_{0}}+\cdots+x_{n}^{a_{n}} \text { com inteiros } a_{i} \geq 2 .
$$

Definição 3.2. Um polinômio $h \in \mathbb{C}\left[x_{0}, \ldots, x_{n}\right]$ é dito um arranjo de Pham-Brieskorn do tipo $\left(r_{0}, \ldots, r_{n} ; d\right)$ se pode ser escrito na forma

$$
h=c f_{1} \cdots f_{p} f_{p+1}^{\alpha_{p+1}} \cdots f_{l}^{\alpha_{l}}
$$

onde $f_{j}=\gamma_{0, j} x_{0}^{\hat{r}_{0} r_{1} \cdots r_{n}}+\cdots+\gamma_{n, j} x_{n}^{r_{0} r_{1} \cdots \hat{r}_{n}}$, com $r_{i}$ e $r_{j}$ coprimos para todo $i \neq j, \alpha_{i}>1$ e $d=$ $\left(p+\sum_{i=p+1}^{l} \alpha_{i}\right) r_{0} \cdots r_{n}$. A notação $\hat{r}_{i}$ significa ausência do termo $r_{i}$ com $i \in\{0, \cdots, n\}$.

Observação 3.3. Observemos aqui que todo o trabalho a ser desenvolvido neste capítulo pode ser aplicado a um caso mais geral onde cada $f_{j}$ é da forma

$$
f_{j}=\gamma_{0, j} x_{0}^{k_{0}\left(\hat{r}_{0} r_{1} \cdots r_{n}\right)}+\cdots+\gamma_{n, j} x_{n}^{k_{n}\left(r_{0} r_{1} \cdots \hat{r}_{n}\right)}
$$

onde os $k_{i}$ são inteiros positivos quaisquer com $i=0, \cdots, n$ e os pesos $r_{i}$ não precisam ser primos entre si mas devem satisfazer a relação $r_{0} \leq \cdots \leq r_{n}$ e de tal forma que as funções $f_{j}$ sejam irredutíveis.

Prosseguimos com o caso anterior simplesmente para simplificar as contas. 
A seguir, descrevemos a técnica desenvolvida por Massey em [17] que reduz uma hipersuperfície com conjunto singular de dimensão arbitrária em uma hipersuperfície com conjunto singular de dimensão um a menos que a anterior e relaciona os números de Lê da primeira com os números de Lê desta hipersuperfície obtida.

\subsection{Raio polar e fórmulas de Lê-Iomdine generalizadas}

As fórmulas de Lê-Iomdine generalizadas descrevem como os números de Lê de uma hipersuperfície singular com conjunto crítico de dimensão $s$ estão relacionados com os números de Lê de uma certa sequência de hipersuperfícies singulares $X_{1}, \cdots, X_{s}$ que se aproxima da singularidade original de tal forma que os conjuntos críticos de seus termos $X_{i}$ tem dimensão $s-i$.

O raio polar é um número associado à curva polar que garante a existência desta sequência de hipersuperfícies.

Suponha que a curva polar $\Gamma_{h, z}^{1}$ é 1-dimensional na origem. Seja $\eta$ uma componente irredutível de $\Gamma_{h, z}^{1}$ (com sua estrutura reduzida).

Definição 3.4. Se $\eta \cap V\left(z_{0}\right)$ é 0-dimensional na origem, definimos o raio polar de $\eta$ (de h em 0 com respeito a z) como sendo $\frac{(\eta \cdot V(h))_{0}}{\left(\eta \cdot V\left(z_{0}\right)\right)_{0}}$.

Se $\eta \cap V\left(z_{0}\right)$ não é 0-dimensional na origem $\left(\eta \subseteq V\left(z_{0}\right)\right)$ então o raio polar de $\eta$ é 1 . Um raio polar (de h em 0 com respeito a z) é o raio polar de qualquer componente da curva polar.

O teorema a seguir fornece as chamadas fórmulas de Lê-Iomdine generalizadas para uma hipersuperfície com conjunto singular qualquer. Essas fórmulas descrevem como os números de Lê de $h$ mudam quando uma grande potência de uma variável é adicionada.

Teorema 3.5. (Fórmulas de Lê-Iomdine generalizadas) Sejam $h:(U, 0) \rightarrow(\mathbb{C}, 0)$ uma função analítica com $U \subseteq \mathbb{C}^{n+1}$ aberto e $s=\operatorname{dim}_{0} \Sigma(h)$ com $s \geq 1$. Considere $z=\left(z_{0}, \ldots, z_{n}\right)$ uma escolha linear de coordenadas tais que $\lambda_{h, z}^{i}(0)$ está definido para todo $i \leq s$. Seja a um número complexo não nulo e use as coordenadas $\widetilde{z}=\left(z_{1}, \ldots, z_{n}, z_{0}\right)$ para $h+a z_{0}^{j}$, onde $j \geq 2$.

Se j é maior ou igual ao raio polar máximo de h, então para todos exceto um número finito de complexos $a$,

i) $\Sigma\left(h+a z_{0}^{j}\right)=\Sigma(h) \cap V\left(z_{0}\right)$ como germes de conjuntos em 0 ; 
ii) $\operatorname{dim}_{0} \Sigma\left(h+a z_{0}^{j}\right)=s-1$;

iii) $\lambda_{h+a z_{0}^{j}, z}^{i}(0)$ existe para todo $i \leq s-1$;

iv) $\lambda_{h+a z_{0}^{j}, \tilde{z}}^{0}(0)=\lambda_{h, z}^{0}(0)+(j-1) \lambda_{h, z}^{1}(0)$

v) $\lambda_{h+a z_{0}^{j}, z^{i}}^{i}(0)=(j-1) \lambda_{h, z}^{i+1}(0)$, para $1 \leq i \leq s-1$.

Além disso, se jé estritamente maior que o raio polar máximo de $h$, as igualdades acima mantêm-se para todo a não nulo. Em particular, esse é o caso se $j \geq 2+\lambda_{h, z}^{0}(0)$.

A demonstração pode ser vista em [17] (Teorema 4.5, página 48).

Observamos que aplicando o teorema indutivamente obtemos, para certos números complexos $a_{0}, \cdots, a_{s}, X_{1}=V\left(h+a_{0} z_{0}^{j_{0}}\right), \cdots, X_{s}=V\left(h+a_{0} z_{0}^{j_{0}}+\cdots+a_{s} z_{s}^{j_{s}}\right)$ onde $j_{i}$ é maior ou igual ao raio polar máximo de $h+a_{0} z_{0}^{j_{0}}, \cdots, z_{i-1}^{j_{i-1}}\left(\right.$ em 0 com respeito a $\left.\tilde{z}=\left(z_{i}, \cdots, z_{n}, z_{0}, \ldots, z_{i-1}\right)\right)$.

Uma importante propriedade das coordenadas pré-polares (Definição 2.8) é que elas são preservadas para cada hipersuperfície da sequência obtida das fórmulas de Lê-Iomdine generalizadas. Este resultado é obtido diretamente aplicando 4.3 e 4.18 de [17].

Corolário 3.6. Se $z=\left(z_{0}, \cdots, z_{n}\right)$ é um sistema de coordenadas pré-polar para $h$ na origem e j é maior ou igual ao raio polar de $h$ (na origem com respeito a $z$ ), então $\tilde{z}=\left(z_{1}, \cdots, z_{n}, z_{0}\right)$ é um sistema de coordenadas pré-polar para $h+a z_{0}^{j}$ na origem, exceto para um número finito de $a \in \mathbb{C}$.

\subsection{Coordenadas pré-polares para arranjos de Pham-Brieskorn}

Para descrever os números de Lê de arranjos de Pham-Brieskorn, primeiro obtemos uma estratificação de $V(h)$ que satisfaz as condições de Whitney, pois com isso esta estratificação é sempre uma boa estratificação de $h$. Na verdade, vamos mostrar que existe uma estratificação minimal da variedade $V(h)$ como definido por Lê e Teissier em [13].

Para finalizar, dado $h$ um arranjo de Pham-Brieskorn do tipo $\left(r_{0}, \ldots, r_{n} ; d\right)$ e assumindo que $r_{0}<\cdots<r_{n}$, obtemos um sistema de coordenadas que é pré-polar para $h$ na origem com relação a estratificação de Whitney minimal da variedade $V(h)$.

Recorremos aqui ao algorítimo de Lê e Teissier [13] para obter tal estratificação. 
De acordo com Lê e Teissier em [13], existe uma sequência de subvariedades algébricas de $V(h), F_{0} \supset F_{1} \supset \cdots \supset F_{k} \cdots$, onde $F_{0}=V(h)$ e $F_{1}$ denota o conjunto singular de $V(h)$. Para obter os outros $F_{j}$ com $j \geq 2$, vamos denotar por $\left(F_{1, j_{1}}\right)_{j_{1} \in J_{1}}$ as componentes de $F_{1}$, então $F_{2}$ é a união do conjunto singular de $F_{1}$ com o conjunto dos pontos em $F_{1, j_{1}}$ tal que a sequência de multiplicidades polares de Lê-Teissier é diferente de uma calculada em um ponto genérico de $F_{1, j_{1}}$.

Aqui é considerada a $k$-ésima multiplicidade polar em um ponto como sendo a multiplicidade da variedade polar $P_{k}\left(V(h), D_{n+1-k}\right)$, definida por Lê e Teissier, neste ponto,

$$
P_{k}\left(V(h), D_{n+1-k}\right)=\overline{\left\{p \in V(h)_{\text {reg }} \mid \operatorname{dim}\left(T_{p} V(h)_{\text {reg }} \cap D_{n+1-k}\right) \geq k\right\}},
$$

onde $D_{n+1-k}$ é um hiperplano genérico e $V(h)_{\text {reg }}$ denota a parte regular de $V(h)$.

Denotemos por $\left(F_{2, j_{2}}\right)_{j_{2} \in J_{2}}$ as componentes irredutíveis de $F_{2}$. Procedendo indutivamente, obtemos que, para $i \geq 2, F_{i}$ é a união do conjunto singular de $F_{i-1}$ com o conjunto dos pontos em $F_{i-1, j_{i-1}}$ tal que a sequência de multiplicidades polares de Lê-Teissier é diferente de uma calculada em um ponto genérico de $F_{i-1, j_{i-1}}$.

A fim de construir a estratificação de Whitney do conjunto $V(h)$, fixamos a seguinte notação: Para todo $i \operatorname{com} 1 \leq i \leq l$ definimos $H_{i}=V\left(f_{i}^{m_{i}}\right) \operatorname{com} m_{i}=1$ se $1 \leq i \leq p$ e $m_{i}=\alpha_{i}$ se $p+1 \leq$ $i \leq l$. Se $I$ denota o conjunto dos índices $\{1, \cdots, l\}$, para qualquer subconjunto $J$ de $I$, definimos

$$
w_{J}:=\bigcap_{j \in J} H_{j} \text { e } S_{J}:=w_{J}-\bigcup_{J \subsetneq K} w_{K} .
$$

Lema 3.7. $\left\{S_{J}\right\}_{J \subseteq I}$ é a estratificação de Whitney minimal de $V(h)$.

Demonstração. Mostramos, para cada $k$, que as componentes irredutíveis $F_{k, j_{k}}$ são da forma

$$
F_{k, j_{k}}=w_{J}:=\bigcap_{j \in J} H_{j} \text { onde } \# J=k+1 .
$$

Como cada função $f_{i}$ tem singularidade isolada na origem, as componentes do conjunto crítico de $V(h)$ são as intersecções $H_{i} \cap H_{j} \operatorname{com} i \neq j$ e $i, j \in I$.

Daí temos que $F_{1}$ é dado pela união das intersecções $H_{i} \cap H_{j} \operatorname{com} i \neq j$ e $i, j \in I$. Em outras palavras, cada $F_{1, j_{1}}$ é da forma $F_{1, j_{1}}=H_{i} \cap H_{j} \operatorname{com} i \neq j$ e $i, j \in I$.

Pela mesma razão, o conjunto crítico de $F_{1}$ tem como componentes as intersecções $H_{i} \cap H_{j} \cap$ $H_{k} \operatorname{com} i, j, k \in I$ distintos. 
Agora, se $p \in V(h)_{\text {reg }}$, então $p \in H_{i}-\bigcup_{j \neq i} H_{j}$ para algum $i$. Desta forma,

$$
\operatorname{dim}\left(T_{p} V(h)_{\text {reg }} \cap D_{n+1-k}\right) \geq k \Rightarrow D_{n+1-k} \subset T_{p} V(h)_{\text {reg }} \Rightarrow \frac{\partial f_{i}}{\partial z_{m}}(p)=0 \text { se } m \geq n+1-k
$$

$$
\begin{aligned}
P_{k}\left(V(h), D_{n+1-k}\right) & =\overline{\bigcup_{i=1}^{l}\left\{p \in H_{i}-\bigcup_{j \neq i} H_{j} \mid \frac{\partial f_{i}}{\partial z_{m}}(p)=0 \text { se } m \geq n+1-k\right\}} \\
& =\bigcup_{i=1}^{l} \overline{\left\{p \in H_{i}-\bigcup_{j \neq i} H_{j} \mid \frac{\partial f_{i}}{\partial z_{m}}(p)=0 \text { se } m \geq n+1-k\right\}} \\
& =\bigcup_{i=1}^{l}\left\{p \in H_{i} \mid \frac{\partial f_{i}}{\partial z_{m}}(p)=0 \text { se } m \geq n+1-k\right\} .
\end{aligned}
$$

Pela descrição anterior, considerando $B_{i}=\left\{p \in H_{i} \mid \frac{\partial f_{i}}{\partial z_{m}}(p)=0\right.$ se $\left.m \geq n+1-k\right\} \mathrm{e}$ $q \in P_{k}\left(V(h), D_{n+1-k}\right)$, temos que

$$
\operatorname{mult}_{q} P_{k}\left(V(h), D_{n+1-k}\right)=\sum_{i, q \in H_{i}} \operatorname{mult}_{q} B_{i}
$$

onde $\operatorname{mult}_{q}$ denota a multiplicidade em $q$.

Portanto, o conjunto de pontos $p \in F_{1, j_{1}}$ tal que a sequência das multiplicidades polares de Lê-Teissier é diferente de uma computada num ponto genérico está contido no conjunto das intersecções da forma $H_{i} \cap H_{j} \cap H_{k} \operatorname{com} i, j, k \in I$ distintos. Deste modo, as componentes de $F_{2}$, $F_{2, j_{2}}$, são as intersecções $H_{i} \cap H_{j} \cap H_{k} \operatorname{com} i, j, k \in I$ distintos.

Procedendo indutivamente obtemos que as componentes de $F_{k}$, denotadas por $F_{k, j_{k}}$, são da forma $w_{J}:=\bigcap_{i \in J} H_{i} \operatorname{com} \# J=k+1$.

Assim, pela Proposição $1.45, S_{J}:=w_{J}-\bigcup_{J \subsetneq K} w_{k}$ é a estratificação de Whitney minimal de $V(h)$, decrita por Lê e Teissier.

O sistema de coordenadas usado até o final deste capítulo será $z=\left(x_{0}, \cdots, x_{n}\right)$. A proposição a seguir garante que $z$ é pré-polar para $h$ em 0 com respeito a estratificação anterior.

Proposição 3.8. O sistema de coordenadas $z=\left(x_{0}, \cdots, x_{n}\right)$ é pré-polar para $h$ com relação a estratificação $\left\{S_{J}\right\}_{J \subseteq I}$. 
Demonstração. A estratificação $\left\{S_{J}\right\}$ é uma boa estratificação para $h$ em 0 pois a $a_{h}$ condição de Thom é uma das condições de uma estratificação de Whitney.

Mostremos que $V\left(x_{0}\right)$ intercepta transversalmente todos os estratos $S_{J} \operatorname{com} S_{J} \neq\{0\}$ e, para todo $j$ tal que $1 \leq j \leq n-1, V\left(x_{j}\right)$ intercepta transversalmente todos os $S_{J} \cap V\left(x_{0}, \cdots, x_{j-1}\right)$ $\operatorname{com} S_{J} \cap V\left(x_{0}, \cdots, x_{j}\right) \neq\{0\}$.

Dizer que $V\left(x_{0}\right)$ intercepta transversalmente $S_{J}$ significa que para todo $p \in V\left(x_{0}\right) \cap S_{J}$ temos que $T_{p} V\left(x_{0}\right)+T_{p} S_{J}=\mathbb{C}^{n+1}$, ou equivalentemente, que o vetor normal a $V\left(x_{0}\right)$ em $p$ não está contido no espaço normal a $S_{J}$ em $p$.

Seja $J=\{i\}$ e considerando $p \in V\left(x_{0}\right) \cap S_{J}$, temos que o vetor normal a $S_{J}$ em $p$ é igual ao vetor normal a $V\left(f_{i}\right)$ em $p$. Agora $v=(1,0, \cdots, 0)$ é o vetor normal de $V\left(x_{0}\right)$ e

$$
w_{i}=\left(0, \gamma_{1, i}\left(r_{0} \hat{r}_{1} \cdots r_{n}\right) \tilde{x}_{1}^{\left(r_{0} \hat{r}_{1} \cdots r_{n}\right)-1}, \cdots, \gamma_{n, i}\left(r_{0} \cdots r_{n-1} \hat{r}_{n}\right) \tilde{x}_{n}^{\left(r_{0} \cdots r_{n-1} \hat{r}_{n}\right)-1}\right)
$$

é o vetor normal de $V\left(f_{i}\right)$ no ponto $\left(0, \tilde{x}_{1}, \cdots, \tilde{x}_{n}\right) \neq(0, \cdots, 0)$ de $V\left(x_{0}\right) \cap S_{J}$.

Claramente $v$ e $w_{i}$ não são paralelos. Logo, $V\left(x_{0}\right)$ intercepta transversalmente $S_{J}$ onde $J=$ $\{i\} \operatorname{com} i=1, \cdots, l$.

Agora, seja $J=\{i, j\}$ com $i \neq j, i, j \in\{1, \cdots, l\}$ e $S_{J} \neq\{0\}$. Temos que o espaço normal a $S_{J}$ em um ponto $(0, \cdots, 0) \neq\left(0, \tilde{x}_{1}, \cdots, \tilde{x}_{n}\right) \in V\left(x_{0}\right) \cap S_{J}$ é gerado pelos vetores

$$
w_{k}=\left(0, \gamma_{1, k}\left(r_{0} \hat{r}_{1} \cdots r_{n}\right) \tilde{x}_{1}^{\left(r_{0} \hat{r}_{1} \cdots r_{n}\right)-1}, \cdots, \gamma_{n, k}\left(r_{0} \cdots r_{n-1} \hat{r}_{n}\right) \tilde{x}_{n}^{\left(r_{0} \cdots r_{n-1} \hat{r}_{n}\right)-1}\right) \operatorname{com} k=i, j
$$

Claramente $v \notin\left[w_{i}, w_{j}\right]$ e, portanto, $V\left(x_{0}\right)$ intercepta transversalmente $S_{J}$.

Com este mesmo raciocínio, prova-se que $V\left(x_{0}\right)$ intercepta transversalmente $S_{J}$ para todo $J$, exceto quando $S_{J}=\{0\}$ e que $V\left(x_{j}\right)$ intercepta transversalmente todos os $S_{J} \cap V\left(x_{0}, \cdots, x_{j-1}\right)$ para todo $1 \leq j \leq n-1 \operatorname{com} S_{J} \cap V\left(x_{0}, \cdots, x_{j-1}\right) \neq\{0\}$.

\subsection{Raio polar de Arranjos de Pham-Brieskorn}

Seja $h \in \mathbb{C}\left[x_{0}, \cdots, x_{n}\right]$ um arranjo de Pham-Brieskorn do tipo $\left(r_{0}, \cdots, r_{n} ; d\right)$ com $r_{0}<\cdots<r_{n}$. Mostremos que o raio polar máximo de $h$ na origem com respeito ao sistema de coordenadas $z=\left(x_{0}, \cdots, x_{n}\right)$ depende somente dos pesos $r_{0}, \cdots, r_{n}$ e do grau de homogeneidade $d$. 
Proposição 3.9. O raio polar máximo de h na origem com respeito ao sistema de coordenadas $z=\left(x_{0}, \cdots, x_{n}\right)$ é menor ou igual a $\frac{d}{r_{0}}$.

Como vimos, $h$ pode ser escrita na forma

$$
h=c f_{1} \cdots f_{p} \cdot f_{p+1}^{\alpha_{p+1}} \cdots f_{l}^{\alpha_{l}}, \text { onde } d=\left(p+\sum_{i=p+1}^{l} \alpha_{i}\right) r_{0} \cdots r_{n} .
$$

Denotemos por $g_{i, j}=f_{1} \cdots \frac{\partial f_{i}}{\partial x_{j}} \cdots f_{l}$ e $g_{j}=g_{1, j}+\cdots+g_{p, j}+\alpha_{p+1} g_{p+1, j}+\cdots+\alpha_{l} g_{l, j}$, com $0 \leq j \leq n$

A fim de calcular o raio polar dos arranjos de Pham-Brieskorn, usamos o seguinte resultado.

Lema 3.10. Para todo $k \in\{0, \cdots, n\}$, os conjuntos $V\left(g_{n}, g_{n-1}, \cdots, g_{k}\right)$ não têm componentes no conjunto crítico de $h$.

Demonstração. Notemos que

$$
g_{j}=\left(r_{0} \cdots \hat{r}_{j} \cdots r_{n}\right) x_{j}^{r_{0} \cdots \hat{r}_{j} \cdots r_{n}-1}\left(\left(m_{1} \gamma_{j, 1}\right) \hat{f}_{1} f_{2} \cdots f_{l}+\cdots+\left(m_{l} \gamma_{j, l}\right) f_{1} \cdots \hat{f}_{l}\right),
$$

onde $\hat{f}_{k}$ significa a ausência da função $f_{k} \operatorname{com} k \in\{1, \cdots, l\}$.

Para obter o conjunto crítico de $h$, como $\frac{\partial h}{\partial x_{j}}=\left(f_{p+1}^{\alpha_{p+1}-1} \cdots f_{l}^{\alpha_{l}-1}\right) g_{j}$, então para todo $i \neq j \in$ $\{0, \cdots, n\}$, consideremos o sistema

$$
\left\{\begin{array}{ll}
m_{1} \gamma_{i, 1} \hat{f}_{1} f_{2} \cdots f_{l}+\cdots & +m_{l} \gamma_{i, l} f_{1} f_{2} \cdots \hat{f}_{l}=0 \\
m_{1} \gamma_{j, 1} \hat{f}_{1} f_{2} \cdots f_{l}+\cdots & +m_{l} \gamma_{j, l} f_{1} f_{2} \cdots \hat{f}_{l}=0
\end{array} .\right.
$$

que é equivalente ao seguinte sistema:

$$
\left\{\begin{array}{ccc}
m_{1} \gamma_{i, 1} \hat{f}_{1} f_{2} \cdots f_{l} & +m_{2} \gamma_{i, 2} f_{1} \hat{f}_{2} \cdots f_{l}+\cdots+m_{l} \gamma_{i, l} f_{1} f_{2} \cdots \hat{f}_{l}=0 \\
0 & +m_{2} k_{2} f_{1} \hat{f}_{2} \cdots f_{l}+\cdots+m_{l} k_{l} f_{1} f_{2} \cdots \hat{f}_{l}=0
\end{array}\right.
$$

onde $k_{m}=\gamma_{j, 1} \gamma_{i, m}-\gamma_{i, 1} \gamma_{j, m}, \operatorname{com} 2 \leq k \leq l$.

Da segunda equação temos que $f_{1}=0$ ou $f_{3} \cdots f_{l}=-\left(\frac{m_{3} k_{3}}{m_{2} k_{2}} f_{2} \hat{f}_{3} \cdots f_{l}+\cdots+\frac{m_{l} k_{l}}{m_{2} k_{2}} f_{2} \cdots \hat{f}_{l}\right)$.

Então

$$
\begin{gathered}
\left(m_{2} \gamma_{i, 2} f_{1}+m_{1} \gamma_{i, 1} f_{2}\right)\left(f_{3} \cdots f_{l}\right)= \\
-\left(m_{2} \gamma_{i, 2} f_{1}+m_{1} \gamma_{i, 1} f_{2}\right)\left(\frac{m_{3} k_{3}}{m_{2} k_{2}} f_{2} \hat{f}_{3} \cdots f_{l}+\cdots+\frac{m_{l} k_{l}}{m_{2} k_{2}} f_{2} \cdots \hat{f}_{l}\right) .
\end{gathered}
$$


Da primeira equação temos que

$$
\left(m_{2} \gamma_{i, 2} f_{1}+m_{1} \gamma_{i, 1} f_{2}\right)\left(f_{3} \cdots f_{l}\right)=f_{1}\left(m_{3} \gamma_{i, 3} f_{2} \hat{f}_{3} \cdots f_{l}+\cdots+m_{l} \gamma_{i, l} f_{2} \cdots \hat{f}_{l}\right) .
$$

Pelo fato de que a decomposição de um polinômio em fatores irredutíveis é única (exceto a ordem) e pela descrição (1) e (2), temos que $f_{3} \cdots f_{l}=0$.

Voltando ao sistema inicial, temos que as soluções são as intersecções $V\left(f_{m}\right) \cap V\left(f_{n}\right)$ com $m \neq n$ em $\{1, \cdots, l\}$. Como $i$ e $j$ são tomados arbitrários, temos que o conjunto crítico (como conjunto) é a união

$$
\bigcup_{i=p+1}^{l} V\left(f_{i}\right) \cup \bigcup_{i \neq j \in\{1, \cdots, p\}} V\left(f_{i}\right) \cap V\left(f_{j}\right) .
$$

Assim, pela descrição dos polinômios $g_{i}$ 's e como cada $f_{i}$ é irredutível, $V\left(g_{n}, g_{n-1}, \cdots, g_{k}\right)$ não tem componentes no conjunto crítico para todo $k \in\{0, \cdots, n\}$.

Pela Proposição 3.8 e pelo Teorema 2.9, para o sistema de coordenadas $z=\left(x_{0}, \cdots, x_{n}\right)$ concluímos que $\Lambda_{h, z}^{i}$ é puramente $i$-dimensional em 0 e os números de Lê $\lambda_{h, z}^{i}(0)$ estão definidos para todo $0 \leq i \leq n$.

Agora, para calcular o raio polar, primeiro precisamos obter a curva polar.

Da Proposição 2.5, para todo $0 \leq i \leq n$,

$$
\left[\Gamma_{h, z}^{i+1}\right] \cdot\left[V\left(\frac{\partial h}{\partial x_{i}}\right)\right]=\left[\Lambda_{h, z}^{i}\right]+\left[\Gamma_{h, z}^{i}\right]
$$

Para $i=n, \sum_{j=p+1}^{l}\left[V\left(f_{j}^{\alpha_{j}-1}\right)\right]+\left[V\left(\frac{\frac{\partial h}{\partial x_{n}}}{\prod f_{j}^{\alpha_{j}-1}}\right)\right]=\left[V\left(\frac{\partial h}{\partial x_{n}}\right)\right]=\Lambda_{h, z}^{n}+\Gamma_{h, z}^{n}$.

Por definição, $\Lambda_{h, z}^{n} \subseteq \Sigma h$ e $\Lambda_{h, z}^{n}$ e $\Gamma_{h, z}^{n}$ são disjuntos. Como $V\left(g_{n}\right)=V\left(\frac{\frac{\partial h}{\partial x_{n}}}{\Pi f_{j}^{\alpha_{j}-1}}\right)$ não está contido no conjunto crítico de $h$, pelo Lema 3.10, obtemos que $\left[\Gamma_{h, z}^{n}\right]=\left[V\left(g_{n}\right)\right]$.

Por indução temos que

$$
\left[\Gamma_{h, z}^{n}\right] \cdot\left[V\left(\frac{\partial h}{\partial x_{n-1}}\right)\right]=\sum_{j=p+1}^{l}\left(\left[V\left(f_{j}^{\alpha_{j}-1}\right)\right] \cdot\left[V\left(g_{n}\right)\right]\right)+\left(\left[V\left(g_{n-1}\right)\right] \cdot\left[V\left(g_{n}\right)\right]\right) .
$$

Como $\left[\Gamma_{h, z}^{n}\right] \cdot\left[V\left(\frac{\partial h}{\partial x_{n-1}}\right)\right]=\Lambda_{h, z}^{n-1}+\Gamma_{h, z}^{n-1}$, usando o mesmo argumento temos $\left[\Gamma_{h, z}^{n-1}\right]=$ $\left[V\left(g_{n-1}, g_{n}\right)\right]$ e, procedendo desta maneira, concluímos que $\left[\Gamma_{h, z}^{1}\right]=\left[V\left(g_{1}, \cdots, g_{n}\right)\right]$. 
Usando novamente o Teorema 2.9 temos que $\Gamma_{h, z}^{1}$ é 1-dimensional. Assim $\Gamma_{h, z}^{1}$ é uma curva algébrica definida sobre $\mathbb{C}$ determinada por $n$ funções polinomiais independentes em $n+1$ variáveis com coeficientes em $\mathbb{C}$. Usando a resultante, nós podemos eliminar todas menos duas das variáveis e reduzir a curva à curva plana equivalente biracional, $\hat{g}(x, y)=0$.

Daí cada componente de $\Gamma_{h, z}^{1}$ tem uma parametrização local. Agora, seja $\eta$ uma componente irredutível de $\Gamma_{h, z}^{1}$ e consideremos $\phi(t)=\left(\phi_{0}(t), \cdots, \phi_{n}(t)\right)$ uma parametrização de $\eta$, onde $\phi_{j}(t)=c_{j} t^{k_{j}}+$ termos de ordem superior.

Demonstração da Proposição 3.9. Vamos supor que $\eta$ intersepta transversalmente $V\left(z_{0}\right)$, pois caso contrário o raio polar é 1. Pela Proposição 1.28,

$$
\left(\eta \cdot V\left(f_{i}\right)\right)_{0}=\text { mult }_{t} f_{i}(\phi(t))=\min _{q}\left\{k_{q}\left(r_{0} \cdots \hat{r}_{q} \cdots r_{n}\right)\right\} \text { e }\left(\eta \cdot V\left(z_{0}\right)\right)_{0}=\text { mult }_{t} z_{0}(\phi(t))=k_{0}
$$

Deste modo,

$$
\frac{(\eta \cdot V(h))_{0}}{\left(\eta \cdot V\left(z_{0}\right)\right)_{0}} \leq \frac{\frac{d}{r_{0} \cdots r_{n}}\left(k_{0}\left(\hat{r}_{0} r_{1} \cdots r_{n}\right)\right)}{k_{0}}=\frac{d}{r_{0}}
$$

e com isso, o raio polar máximo de $h$ é menor ou igual a $\frac{d}{r_{0}}$.

Mostraremos agora que também podemos calcular o raio polar da função $h+a_{0} x_{0}^{\frac{d}{r_{0}}}$ em termos dos pesos e grau.

Proposição 3.11. O raio polar máximo de $h+a_{0} x_{0}^{\frac{d}{r_{0}}}$ na origem com respeito ao sistema de coordenadas $z=\left(x_{1}, \cdots, x_{n}, x_{0}\right)$ é menor ou igual a $\frac{d}{r_{1}}$.

$O$ raio polar máximo de $h+a_{0} x_{0}^{\frac{d}{r_{0}}}+\cdots+a_{i-1} x_{i-1}^{\frac{d}{r_{i-1}}}$ na origem com respeito ao sistema de coordenadas $z=\left(x_{i}, \cdots, x_{n}, x_{0}, \cdots, x_{i-1}\right)$ é menor ou igual a $\frac{d}{r_{i}}$ para todo $i=1, \cdots, n-1$.

Demonstração. Como $z=\left(x_{0}, \cdots, x_{n}\right)$ é pré-polar para $h$ em 0 então, pela Proposição 3.6, $\tilde{z}=\left(x_{1}, \cdots, x_{n}, x_{0}\right)$ é pré-polar para $h+a_{0} x_{0}^{\frac{d}{r_{0}}}$ em 0 , exceto para um número finito de $a_{0}$. Consideremos $a_{0}$ que satisfaça esta condição. Assim, temos que a curva polar de $h+a_{0} x_{0}^{\frac{d}{r_{0}}}$ com relação a $\tilde{z}$ é puramente 1-dimensional em 0 e, pela técnica da Proposição 2.5, ela é determinada por $n$ funções polinomiais independentes em $n+1$ variáveis com coeficientes em $\mathbb{C}$. Daí, qualquer componente irredutível desta curva polar tem uma parametrização.

Seja $\eta$ uma componente irredutível da curva polar de $h+a_{0} x_{0}^{\frac{d}{r_{0}}}$ com relação a $\tilde{z}$ que intersepta transversalmente $V\left(x_{1}\right)$ e $\phi(t)=\left(\phi_{0}(t), \cdots, \phi_{n}(t)\right)$ uma parametrização de $\eta$, onde 
$\phi_{q}(t)=c_{q} t^{k_{q}}+$ termos de ordem superior.

Sabemos que $\left(\eta \cdot V\left(h+a_{0} x_{0}^{\frac{d}{r_{0}}}\right)\right)_{0}=\operatorname{mult}_{t}\left(h+a_{0} x_{0}^{\frac{d}{r_{0}}}\right)(\phi(t))=\operatorname{mult}_{t}\left(h(\phi(t))+a_{0} x_{0}^{\frac{d}{r_{0}}}(\phi(t))\right)=$ $\operatorname{mult}_{t} h(\phi(t))=\min _{q}\left\{k_{q}\left(r_{0} \cdots \hat{r}_{q} \cdots r_{n}\right)\right\}$.

Portanto,

$$
\frac{\left(\eta \cdot V\left(h+x_{0}^{\frac{d}{r_{0}}}\right)\right)_{0}}{\left(\eta \cdot V\left(x_{1}\right)\right)_{0}} \leq \frac{\frac{d}{r_{0} \cdots r_{n}}\left(k_{1}\left(r_{0} \hat{r}_{1} \cdots r_{n}\right)\right)}{k_{1}}=\frac{d}{r_{1}} .
$$

Deste modo o raio polar máximo de $h+a_{0} x_{0}^{\frac{d}{r_{0}}}\left(\mathrm{em} 0\right.$ com respeito a $\left.\left(x_{1}, \cdots, x_{n}, x_{0}\right)\right)$ é menor ou igual a $\frac{d}{r_{1}}$.

Procedendo de maneira análoga, o raio polar máximo de $h+a_{0} x_{0}^{\frac{d}{r_{0}}}+\cdots+a_{i-1} x_{i-1}^{\frac{d}{r_{i-1}}}$ (em 0 com respeito a $\left.\left(x_{i}, \cdots, x_{n}, x_{0}, \cdots, x_{i-1}\right)\right)$ é menor ou igual a $\frac{d}{r_{i}}$ para todo $i=1, \cdots, n-1$.

\subsection{Caracterização dos números de Lê}

Agora vamos caracterizar os números de Lê na origem de um arranjo de Pham-Brieskorn $h$ em $\mathbb{C}\left\{x_{0}, \cdots, x_{n}\right\}$ do tipo $\left(r_{0}, \cdots, r_{n}, d\right)$ com $r_{0}<\cdots<r_{n}$ na origem com respeito a $z=$ $\left(x_{0}, \cdots, x_{n}\right)$ somente utilizando o número de variáveis, os pesos e o grau de homogeneidade.

No que segue exibimos a fórmula chave para fazer esta descrição, que é um tipo de fórmula de Plücker.

Proposição 3.12. Seja h um arranjo de Pham-Brieskorn do tipo $\left(r_{0}, \ldots, r_{n} ; d\right)$ nas variáveis $x_{0}, \cdots, x_{n}$. Então, para o sistema de coordenadas $z=\left(x_{0}, \cdots, x_{n}\right)$,

$$
\sum_{i=0}^{n}\left(\prod_{k=0}^{i-1}\left(\frac{d}{r_{k}}-1\right)\right) \lambda_{h, z}^{i}(0)=\left(\frac{d}{r_{0}}-1\right) \cdots\left(\frac{d}{r_{n}}-1\right) .
$$

Demonstração. Da Proposição 3.9, temos que o raio polar máximo de $h$ na origem com respeito ao sistema de coordenadas $z=\left(x_{0}, \cdots, x_{n}\right)$ é menor ou igual a $\frac{d}{r_{0}}$. Então consideremos $j_{0}=\frac{d}{r_{0}}$ para aplicar o Teorema 3.5 e obter que existe $a_{0} \in \mathbb{C}^{*}$, tal que $h+a_{0} x_{0}^{\frac{d}{r_{0}}}$ tem conjunto crítico de dimensão $n-1$.

Aplicando agora a Proposição 3.11, para todos exceto um número finito de $a_{i} \in \mathbb{C}^{*}$, o raio polar máximo de $h+a_{0} x_{0}^{\frac{d}{r_{0}}}+\cdots+a_{i-1} x_{i-1}^{\frac{d}{r_{i-1}}}$, na origem com relação ao sistema de coordenadas $\tilde{z}=\left(x_{i}, \cdots, x_{n}, x_{0}, \cdots, x_{i-1}\right)$, é menor ou igual a $\frac{d}{r_{i}}$ para todo $1 \leq i \leq n-1$. 
Assim, aplicando o Teorema 3.5 indutivamente com $j_{i}=\frac{d}{r_{i}}, 1 \leq i \leq n-1$ obtemos que existem $a_{1}, \cdots, a_{n-1} \in \mathbb{C}^{*}$ tal que $f:=h+a_{0} x_{0}^{j_{0}}+a_{1} x_{1}^{j_{1}}+\cdots+a_{n-1} x_{n-1}^{j_{n-1}}$ tem singularidade isolada na origem e

$$
\mu(f)=\sum_{i=0}^{n}\left(\prod_{k=0}^{i-1}\left(\frac{d}{r_{k}}-1\right)\right) \lambda_{h, z}^{i}(0) .
$$

Como $f$ é um polinômio quase homogêneo do tipo $\left(r_{0}, \cdots, r_{n} ; d\right)$, concluímos do Teorema 1 de $[18]$ que $\mu(f)=\frac{\left(d-r_{0}\right) \cdots\left(d-r_{n}\right)}{r_{0} \cdots r_{n}}$.

Assim,

$$
\sum_{i=0}^{s}\left(\prod_{k=0}^{i-1}\left(\frac{d}{r_{k}}-1\right)\right) \lambda_{h, z}^{i}(0)=\left(\frac{d}{r_{0}}-1\right) \cdots\left(\frac{d}{r_{n}}-1\right) .
$$

Observação 3.13. Observamos que Massey mostra (em [17]-Corollary 4.7) uma fórmula de Plücker para polinômios homogêneos de grau d em $n+1$ variáveis dada como:

"Se $\lambda_{h, z}^{i}(0)$ existe para todo $i \leq s$, onde $s=\operatorname{dim} \Sigma h$, então $\sum_{i=0}^{s}(d-1)^{i} \lambda_{h, z}^{i}(0)=(d-1)^{n+1}$ ".

Massey em [17] (Capítulo 5) usa esta fórmula para caracterizar cada número de Lê (na origem) de um arranjo linear.

Agora vamos utilizar a fórmula obtida na proposição anterior para caracterizar cada número de Lê (na origem) de um arranjo de Pham-Brieskorn.

Lema 3.14. $\Lambda_{h, z}^{k}=\sum_{\operatorname{dim} S_{J}=k} a_{J}\left[w_{J}\right]$, com $a_{J}=\lambda_{h_{\left.\right|_{N}}, z}^{k}(p)$ para algum $p \in S_{J} \operatorname{com} p=\left(p_{0}, \cdots, p_{n}\right)$ $e N=V\left(x_{0}-p_{0}, \cdots, x_{k-1}-p_{k-1}\right)$.

Demonstração. Primeiramente vamos ver que, como conjuntos, os ciclos de Lê de $h$ com relação ao sistema de coordenadas $z=\left(x_{0}, \cdots, x_{n}\right)$ são dados pela união dos $w_{J}$ de dimensão correta.

Vamos descrevê-los indutivamente. Por definição $\Lambda_{h, z}^{n}$ é formado pelas componentes de $V\left(\frac{\partial h}{\partial x_{n}}\right)$ que estão contidas no conjunto crítico de $h$.

Como vimos, $\frac{\partial h}{\partial x_{n}}=\left(f_{p+1}^{\alpha_{p+1}-1} \cdots f_{l}^{\alpha_{l}-1}\right) g_{n}$ e $V\left(g_{n}\right)$ não têm componentes contidas no conjunto crítico. Assim $\Lambda_{h, z}^{n}$ é, como conjunto, a união dos $V\left(f_{i}\right) \operatorname{com} i=p+1, \cdots, l$.

Agora, consideramos a interseção de $V\left(\frac{\partial h}{\partial x_{n-1}}\right)$ com as componentes de $V\left(\frac{\partial h}{\partial x_{n}}\right)$ que não estão no conjunto crítico de $h$. Temos que $\Lambda_{h, z}^{n-1}$ é formado pelas componentes desta interseção que estão contidas no conjunto crítico de $h$. 
Como vimos, $\frac{\partial h}{\partial x_{n-1}}=\left(f_{p+1}^{\alpha_{p+1}-1} \cdots f_{l}^{\alpha_{l}-1}\right) g_{n-1}$ e $V\left(g_{n-1}, g_{n}\right)$ não têm componentes contidas no conjunto crítico. Assim $\Lambda_{h, z}^{n-1}$ é, como conjunto, a união das interseções $V\left(f_{i}\right) \cap V\left(f_{j}\right)$ com $i \neq j, i=p+1, \cdots, l$ e $j=1, \cdots, l$. O resultado segue procedendo indutivamente.

Assim, como ciclos, para todo $k, \Lambda_{h, z}^{k}=\sum_{\operatorname{dim} S_{J}=k} a_{J}\left[w_{J}\right]$, para algum $a_{J}$.

Seja $\tilde{J} \subseteq I$ tal que a dimensão de $S_{\tilde{J}}$ é $k$. Consideremos $p=\left(p_{0}, \cdots, p_{n}\right) \in S_{\tilde{J}}$.

Como conjuntos, temos que

$$
\begin{aligned}
\Sigma\left(h_{\mid V\left(x_{0}-p_{0}, \ldots, x_{k-1}-p_{k-1}\right)}\right) & =V\left(x_{0}-p_{0}, \ldots, x_{k-1}-p_{k-1}, \frac{\partial h}{\partial z_{k}}, \ldots, \frac{\partial h}{\partial z_{n}}\right) \\
& =V\left(x_{0}-p_{0}, \ldots, x_{k-1}-p_{k-1}\right) \cap\left(\Sigma(h) \cup \Gamma_{h, z}^{k}\right) .
\end{aligned}
$$

Como $\Lambda_{h, z}^{k}$ e $\Gamma_{h, z}^{k}$ são disjuntos, temos que $p \notin \Gamma_{h, z}^{k}$. Assim, pelo Teorema 2.9, $V\left(x_{0}-p_{0}, \ldots, x_{k-1}-p_{k-1}\right) \cap \Gamma_{h, z}^{k}$ é vazio em $p$.

Portanto, considerando $N=V\left(x_{0}-p_{0}, \cdots, x_{k-1}-p_{k-1}\right)$, temos que

$$
\Sigma\left(h_{\mid N}\right)=\Sigma(h) \cap N \text { em } p
$$

Usando a Proposição 2.6, $\lambda_{h_{\mid},}^{k}, \tilde{z}(p)=\lambda_{h, z}^{k}(p)$, onde $\tilde{z}=\left(x_{k}, \cdots, x_{n}, x_{0}, \cdots, x_{k-1}\right)$.

Por outro lado, o ciclo $\Lambda_{h, z}^{k} \cdot V\left(x_{0}-p_{0}, \cdots, x_{k-1}-p_{k-1}\right)$ é da forma

$$
\sum_{\operatorname{dim} S_{J}=k} a_{J}\left(\left[w_{J}\right] \cdot V\left(x_{0}-p_{0}, \cdots, x_{k-1}-p_{k-1}\right)\right) .
$$

Agora, $\left[w_{J}\right] \cdot V\left(x_{0}-p_{0}, \cdots, x_{k-1}-p_{k-1}\right)=[p]$ se $J=\tilde{J}$.

Deste modo, $\left(\Lambda_{h, z}^{k} \cdot V\left(x_{0}-p_{0}, \cdots, x_{k-1}-p_{k-1}\right)\right)_{p}=a_{\tilde{J}}$. Portanto, $a_{\tilde{J}}=\lambda_{h_{\mid}, z}^{k}(p)$.

Observação 3.15. Considerando a translação $\left(x_{0}, \cdots, x_{n}\right) \stackrel{\phi}{\rightarrow}\left(X_{0}, \cdots, X_{n}\right)$ onde $X_{i}=x_{i}-p_{i}$, que leva p na origem, temos que $h_{\left.\right|_{N}}$ em p no novo sistema de coordenadas pode ser visto numa vizinhança da origem como sendo $\prod_{j=1}^{l} \tilde{f}_{j}^{m_{j}} \operatorname{com} \tilde{f}_{j}=\phi\left(f_{i_{x_{0}=\cdots=x_{k-1}=0}}\right)$.

Temos que $p \in S_{J}$. Como $p \notin V\left(f_{j}\right)$ se $j \notin J$, então $0 \notin V\left(\tilde{f}_{j}\right)$ se $j \notin J$. Assim, numa vizinhança da origem $\prod_{j \notin J} \tilde{f}_{j}^{m_{j}}$ é uma unidade e com isso temos que $h_{\left.\right|_{N}}$ pode ser visto, numa vizinhança da origem, como sendo $\prod_{j \in J} \tilde{f}_{j}^{m_{j}}$. 
Para cada hipersuperfície $H_{i}$, denominamos de multiplicidade pesada de $H_{i}$, mult $H_{P}$, o número $m_{i}\left(r_{0} \cdots r_{n}\right)$. Para simplificar as notações, escreveremos simplesmente $H$ para indicar as hipersuperfícies que definem $V(h), w$ ou $v$ suas possíveis intersecções e $\mathscr{A}$ a coleção de todos estes $w$.

$$
\operatorname{Denotemos} e(w):=\sum_{w \subseteq H} \operatorname{mult}_{\mathrm{P}} H \text { e } k(w):=\frac{\left(\frac{e(w)}{r_{0}}-1\right) \cdots\left(\frac{e(w)}{r_{n}}-1\right)}{\prod_{i=0}^{\operatorname{dim} w-1}\left(\frac{e(w)}{r_{i}}-1\right)} .
$$

Definição 3.16. Indutivamente sobre a dimensão de $w$, definimos

$$
\eta(w):=k(w)-\sum_{v \supseteq w} \eta(v)\left(\prod_{i=1}^{\operatorname{codim}_{v} w}\left(\frac{e(w)}{r_{n-i}}-1\right)\right)
$$

Teorema 3.17. Seja h um arranjo de Pham-Brieskorn como definido anteriormente. Então, para o sistema de coordenadas $z=\left(x_{0}, \cdots, x_{n}\right)$,

$$
\lambda_{h, z}^{i}(0)=\sum_{w \in \mathscr{A}, \operatorname{dim} w=i} \eta(w)
$$

Demonstração. Pelo Lema 3.14 e pela Observação $3.15, \Lambda_{h, z}^{k}=\sum_{\operatorname{dim} S_{J}=k} a_{J}\left[w_{J}\right]$, com $a_{J}=$ $\lambda_{g_{J}, z}^{0}(0)$ onde $g$ é o produto de polinômios do tipo Pham-Brieskorn em $n+1-k$ variavéis, mantendo os pesos das variáveis que ficaram, e grau de homogeneidade $e\left(w_{J}\right)$.

Deste modo, $\lambda_{h, z}^{n}(0)=\sum_{\operatorname{dim} S_{J}=n} a_{J}=\sum_{\operatorname{dim} S_{J}=n} \lambda_{g_{J}, z}^{0}(0)$, onde cada $g_{J}$ é um polinômio em uma variável com conjunto crítico de dimensão $0, J=\{i\}, 1, \cdots, l$ e grau $e\left(w_{\{i\}}\right)$.

Aplicando a Proposição 3.12 para $g_{i}$ na origem com relação ao sistema de coordenadas $z=\left(X_{n}\right)$, temos que $\lambda_{g_{\{i\}}, z}^{0}(0)=\frac{e\left(w_{\{i\}}\right)}{r_{n}}-1=\eta\left(w_{\{i\}}\right)$. Portanto,

$$
\lambda_{h, z}^{n}(0)=\sum_{i=1}^{l} \eta\left(w_{\{i\}}\right)=\sum_{\operatorname{dim} v=n} \eta(v) .
$$

Da mesma maneira temos que $\lambda_{h, z}^{n-1}(0)=\sum_{\operatorname{dim} S_{J}=n-1} a_{J}=\sum_{\operatorname{dim} S_{J}=n-1} \lambda_{g_{J}, z}^{0}(0)$, onde cada $g_{J}$ é um polinômio em duas variáveis com conjunto crítico de dimensão 1 de grau $e\left(w_{J}\right)$.

Aplicando a Proposição 3.12 para $g_{J}$ na origem com relação ao sistema de coordenadas 
$z=\left(X_{n-1}, X_{n}\right)$, temos que

$$
\lambda_{g_{\{J\}}, z}^{0}(0)+\left(\frac{e\left(w_{J}\right)}{r_{n-1}}-1\right) \lambda_{g_{\{J\}}, z}^{1}(0)=\left(\frac{e\left(w_{J}\right)}{r_{n-1}}-1\right)\left(\frac{e\left(w_{J}\right)}{r_{n}}-1\right) .
$$

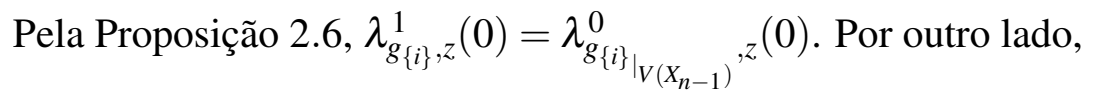

$$
\lambda_{\left.g_{\{i\}}\right|_{V\left(X_{n-1}\right)}, z}^{0}(0)=\sum_{w_{\tilde{J} \supsetneq} \supsetneq w_{J}, \operatorname{dim} S_{\tilde{J}}=n} \lambda_{h_{\left.\right|_{\tilde{J}}}, z}^{0}\left(p_{\tilde{J}}\right)
$$

$\operatorname{com} p_{\tilde{J}}=\left(p_{\tilde{J}, 1}, \cdots, p_{\tilde{J}, n}\right)$ e $N_{\tilde{J}}=\left(x_{0}-p_{\tilde{J}, 0}, \cdots, x_{\tilde{J}, n-1}-p_{\tilde{J}, n-1}\right)$

Novamente aplicando a Observação $3.15, h_{\left.\right|_{\tilde{J}}}$ numa vizinhança de $p_{\tilde{J}}$ pode ser visto, por mudança de coordenadas, como um polinômio (numa vizinhança da origem) em uma variável com conjunto crítico de dimensão $0, \tilde{J}=\{i\}, 1, \cdots, l$ e grau $e\left(w_{\{i\}}\right)$. Pelo caso anterior, temos que $\lambda_{h_{\left.\right|_{\tilde{J}}}, z}^{0}\left(p_{\tilde{J}}\right)=\eta\left(w_{\tilde{J}}\right)$.

Assim, $\lambda_{g_{\{i\}}, z}^{1}(0)=\sum_{w_{J} \supsetneq w, \operatorname{dim} S_{J}=n} \eta(w)$ e com isso

$$
\lambda_{g_{\{J\}}, z}^{0}(0)=\left(\frac{e\left(w_{J}\right)}{r_{n-1}}-1\right)\left(\frac{e\left(w_{J}\right)}{r_{n}}-1\right)-\sum_{w_{J} \supsetneq w, \operatorname{dim} S_{J}=n}\left(\frac{e\left(w_{J}\right)}{r_{n-1}}-1\right) \eta(w) .
$$

Portanto, $\lambda_{h, z}^{n-1}(0)=\sum_{\operatorname{dim} v=n-1} \eta(v)$.

Procedendo indutivamente obtemos $\lambda_{h, z}^{i}(0)=\sum_{\operatorname{dim} v=i} \eta(v)$, com $i=1, \cdots, n$.

Para obter $\lambda_{h, z}^{0}(0)$ basta substituir cada $\lambda_{h, z}^{i}(0)=\sum_{\operatorname{dim} v=i} \eta(v)$. com $i=1, \cdots, n$ na fórmula para $w=\bigcap_{i=1}^{l} H_{i}$ 


\section{- \\ Classes Características para Variedades Singulares}

Em 1935, Stiefel e Whitney construíram independentemente, classes características para variedades suaves, ou melhor, associadas a fibrados vetoriais. Em seu artigo [5] de 1946, Chern deu várias definições equivalentes para classes características sobre variedades hermitianas, a partir de então chamadas de classes de Chern. Assim, no caso de fibrados vetoriais complexos, estas classes são chamadas de classes de Chern e no caso real de classes de Stiefel-Whitney.

O primeiro trabalho que surgiu no intuito de generalizar as classes de Chern para o caso de variedades singulares foi feito por M. H. Schwartz em [26]. Depois disso, surgiram pelo menos mais duas maneiras de obter esta generalização. Neste capítulo vamos descrever duas delas, a generalização introduzida por MacPherson em [16] e a definida por Fulton e Johnson em [8].

Em 1981, Brasselet e Schwartz ([2]) mostraram a existência de um isomorfismo (isomorfismo de Alexander) entre as classes de Schwartz e as de MacPherson (Observação 4.9) e desde então estas são chamadas de classes de Schwartz-MacPherson.

Observemos que a diferença entre as classes de Schwartz-MacPherson e as classes de FultonJohnson é um dos principais objetos de estudo deste trabalho, cujos resultados aparecem no capítulo a seguir.

\subsection{Classes de Chern de um fibrado}

A referência para detalhes é [21].

As classes de Chern aparecem como resposta à seguinte pergunta: Dado um fibrado vetorial 
(complexo) $\xi=(E, B, \pi)$ de dimensão $n$, é possível construir $k$ seções linearmente independentes deste fibrado onde $1 \leq k \leq n$ ?

Para cada fibra $F$ de $\xi$, vamos considerar a variedade de Stiefel $V_{k}(F)$ consistindo de todas as $k$-uplas de vetores linearmente independentes de $F$. Denotamos por $V_{k}(\xi)$ o fibrado com base $B$ e fibra típica $V_{k}(F)$. Uma seção deste fibrado representa $k$ seções linearmente independentes de $\xi$.

Por outro lado, $B$ admite uma triangulação $(k)$ de tal forma que todo simplexo $\sigma$ de $(K)$ está num aberto $U$ da trivialização local de $V_{k}(\xi)$, Suponha que exista uma seção $v^{k}$ de $V_{k}(\xi)$ definida em $\partial \sigma$, o bordo de um simplexo de dimensão $j$. Como o aberto $U$ satisfaz a trivialização local, temos que $V_{k}(\xi)_{\mid} \simeq U \times V_{k}(F)$. Sendo $p_{2}$ a projeção sobre o segundo fator, temos a seguinte composição:

$$
\partial \sigma \stackrel{v^{k}}{\rightarrow} V_{k}(\xi)_{\left.\right|_{U}} \simeq U \times V_{k}(F) \stackrel{p_{2}}{\rightarrow} V_{k}(F)
$$

que nos dá uma aplicação de $S^{j-1} \simeq \partial \sigma \rightarrow V_{k}(F)$. Portanto temos um elemento $\left[\gamma\left(v^{k}, \sigma\right)\right]$ do grupo de homotopia $\pi_{j-1}\left(V_{k}(F)\right.$ ). Estes grupos foram calculados por Stiefel e Whitney (em [24]), que obtiveram a seguinte descrição

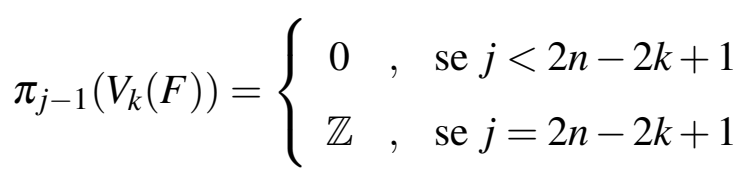

Assim, não existe obstrução à construção de $k$-referenciais sobre os esqueletos de dimensão menor que $2(n-k+1)$.

Deste modo sempre temos um frame definido sobre o bordo de um simplexo $\sigma$ de dimensão $2 p=2(n-k+1)$ e vimos que este define um elemento $\left[\gamma\left(v^{k}, \sigma\right)\right] \in \pi_{2 p-1}\left(V_{k}(F)\right) \simeq \mathbb{Z}$.

Pode-se estender o frame $v$, definido em $\partial \sigma$, através de uma homotetia centrada no baricentro de $\sigma$, que denotamos por $\hat{\sigma}$, com singularidade isolada no mesmo. Calculamos o índice do frame $v$ estendido, $I(v, \hat{\sigma})$, cujo valor é $I(v, \hat{\sigma})=\left[\gamma\left(v^{k}, \sigma\right)\right] \in \mathbb{Z}$.

Definimos assim a cocadeia

$$
[\gamma(v)]=\sum_{\operatorname{dim}(\sigma)=2 p} I(v, \hat{\sigma}) \sigma^{*}
$$

onde $\sigma^{*}$ é a cocadeia cujo valor é 1 sobre a cadeia $\sigma$ e 0 para as demais. 
Esta cocadeia é um cociclo, denominado cociclo de obstrução, e sua classe é denominada de $p$-ésima classe de Chern de $\xi$ e denotada por $c^{p}(\xi)$.

O termo classe total de Chern do fibrado $\xi$ de dimensão $n$ e base $B$, denotado por $c(\xi)$, é a soma formal $1+c^{1}(\xi)+\cdots+c^{n}(\xi)$ no anel $H^{*}(B)$.

Quando $\xi$ é o fibrado tangente de $B$, a classe do ciclo de obstrução é denominada de $p$-ésima classe de Chern de $B$ e denotada por $c^{p}(B)$. A classe total de Chern de $B$ é, como anteriormente, a soma formal $1+c^{1}(B)+\cdots+c^{n}(B)$ no anel $H^{*}(B)$ com $n$ a dimensão de $B$.

\subsection{Classes de Schwartz-MacPherson}

As classes definidas por MacPherson foram definidas utilizando o fibrado de Nash, generalizando no sentido axiomático as classes de Chern com uma prova da conjectura de Deligne e Grothendieck sobre a existência e unicidade de classes características de variedades singulares.

As referências para detalhes são [16] e [3].

\subsubsection{Conjectura de Deligne-Grothendieck}

Para compreendermos o que diz a conjectura de Deligne-Grothendieck, necessitamos definir conjuntos e funções construtíveis.

Definição 4.1. Um conjunto construtível em uma variedade algébrica $V$ é aquele que pode ser obtido de subvariedades algébricas de $V$ através de um número finito de operações de união e complementação.

Definição 4.2. Uma função construtível $\alpha: V \rightarrow \mathbb{Z}$ é aquela em que $V$ admite uma partição finita em subconjuntos construtíveis tais que $\alpha$ é constante em cada um deles. Assim, se $Y_{i}$, $i=1, \cdots, n$, são os tais subconjuntos construtíveis de $V$, e $1_{\left.\right|_{i}}$ é a função característica de $Y_{i}$, então $\alpha$ pode ser escrita como:

$$
\alpha=\sum_{i=1}^{n} \alpha_{i} 1_{\left.\right|_{i}}
$$

onde $\alpha_{i} \in \mathbb{Z}, i=1, \cdots, n$, são os valores assumidos pela função $\alpha$.

Considere o funtor covariante $F$ (obtido em [16]-Proposição 1) da categoria das variedades algébricas compactas $V$ na categoria onde cada objeto $F(V)$ é o grupo das funções construtíveis 
em $V$ e para cada morfismo $f: V \rightarrow W$, o morfismo associado é o homomorfismo $f_{*}: F(V) \rightarrow$ $F(W)$ tal que, para todo fechado $Y$ de $V$,

$$
f_{*}\left(1_{\left.\right|_{Y}}\right)(w)=\chi\left(f^{-1}(w) \cap Y\right) .
$$

Dado $\alpha \in F(V)$, define-se $f_{*}(\alpha)$ da seguinte forma. Suponha $\left\{S_{j}\right\}$ uma estratificação de $V$ subordinada a $\alpha$ e a $f$. Assim

$$
f_{*}(\alpha)(w)=\sum_{j} \alpha\left(S_{j}\right) \chi\left(f^{-1}(w) \cap S_{j}\right)
$$

O seguinte teorema é a Conjectura feita por Deligne e Grothendieck.

Teorema 4.3. ([16]-Teorema 1) Dada qualquer variedade algébrica compacta $V$, para toda função construtível $\alpha$ em $V$ existe uma única classe $c_{*}(\alpha) \in H_{*}(V)$ satisfazendo:

i) $c_{*}(\alpha+\beta)=c_{*}(\alpha)+c_{*}(\beta)$

ii) Para todo morfismo $f: V \rightarrow W$, temos $f_{*}\left[c_{*}(\alpha)\right]=c_{*}\left(f_{*}(\alpha)\right)$, onde, no primeiro lado da igualdade, $f_{*}$ indica o homomorfismo induzido por $f$ em homologia;

iii) Se $V$ for uma variedade suave, então $c_{*}\left(1_{\left.\right|_{V}}\right)=c(V) \cap[V]=$ dual $c(V)$ (Dualidade de Poincaré).

Observação 4.4. A hipótese de compacidade pode ser retirada e considerando-se aplicações próprias e a homologia de Borel-Moore.

\subsubsection{Blow-up de Nash}

Com o intuito de provar o Teorema 4.3, MacPherson construiu classes que solucionam tal conjectura cuja definição utiliza o conceito de obstrução local de Euler e, que por sua vez, é descrito utilizando o Blow-up de Nash.

Seja $X$ uma variedade analítica de dimensão $n$, que admite uma estratificação de Whitney, mergulhada em uma variedade suave $M$ de dimensão $m$. Consideremos o fibrado em Grassmaniannas do $n$-planos (complexos) de $T M$, denotado por $\tilde{M}$, cuja fibra em $x \in M$ é o conjunto $G_{n}\left(T_{x} M\right) \simeq G_{n}\left(\mathbb{C}^{m}\right)$, que é a grassmanianna dos $n$-planos em $\mathbb{C}^{m}$.

Denotemos por $v: \tilde{M} \rightarrow M$ a projeção deste fibrado. Sobre a parte regular $X_{r e g}$ de $X$, existe 
uma seção $s$ deste fibrado, dada por $s(x):=\left(x, T_{x} X_{r e g}\right)$, já que $T_{x} X_{\text {reg }}$ consiste de um $n$-plano que é subespaço de $T_{x} M$.

Definição 4.5. A modificação de Nash X̃ de X é definida como sendo o fecho da imagem de $s$ em $\tilde{M}$.

Denotemos também por $v$ a projeção induzida pela restrição a $\tilde{X}$.

O fibrado tautológico $T$ sobre $\tilde{M}$ é definido da seguinte forma: a fibra $T_{p}$ de $T$ em um ponto $p=(x, P) \in \tilde{M}$ é o conjunto de vetores $v$ do $n$-plano $P$. Definimos o fibrado $\tilde{T}$ com base $\tilde{X}$ como a restrição de $T$ sobre $\tilde{X}$. Este fibrado é chamado fibrado de Nash de $X$.

O seguinte diagrama sistematiza bem toda esta construção.

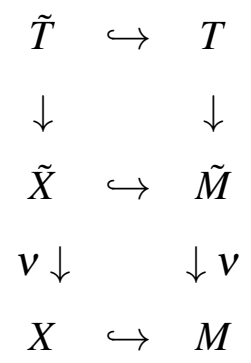

\subsubsection{Obstrução local de Euler}

Seja $p \in X$. Numa vizinhança de $p$ em $M$, considere as coordenadas locais $z=\left(z_{1}, \cdots, z_{n}\right)$ tais que $z(p)=0$. Considere também a função a valores reais $\lambda(z)=\|z\|^{2}=z_{1} \bar{z}_{1}+\cdots+z_{n} \bar{z}_{n}$.

Para todo $p^{\prime}$ suficientemente próximo de $p$ temos que $d_{p^{\prime}} \lambda \in\left(T_{p^{\prime}} M\right)^{*}$, onde $\left(T_{p^{\prime}} M\right)^{*}$ denota o espaço dual de $T_{p^{\prime}} M$, isto é, o espaço das transformações lineares em $T_{p^{\prime}} M$. Então $d \lambda$ é uma seção local de $(T M)^{*}$.

Temos o seguinte diagrama:

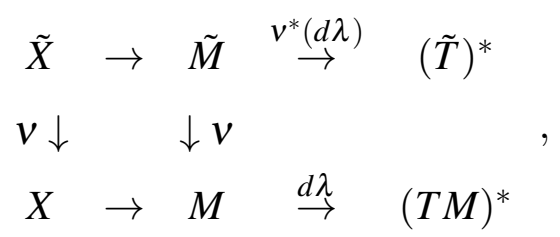

onde $v^{*}(d \lambda)$ é o fibrado induzido por $d \lambda$ definido da seguinte maneira: considere $x \in M$ sufi- 
cientemente próximo de $p$ e $\tilde{x}$ tal que $v(\tilde{x})=x$. Como $\tilde{x}$ é um subespaço de $T_{x} M$, definimos

$$
v^{*}(d \lambda)(\tilde{x}):=(d \lambda)(v(\tilde{x}))_{\mid \tilde{x}}: \tilde{x} \rightarrow \mathbb{R} \in(\tilde{x})^{*}
$$

Assim $v^{*}(d \lambda)$ é uma seção local do dual do fibrado tautológico sobre $M$ e, restringindo $v^{*}(d \lambda)$ a $\tilde{X}$, obtemos uma seção local de $(\tilde{E})^{*}$ que denotamos por $r$. Se $\varepsilon$ é pequeno, esta seção é não nula em $v^{-1}\left(S_{\varepsilon}\right)$. Logo, existe um cociclo de obstrução

$$
\left.E u(\tilde{E}, r) \in H^{2 n}\left(v^{-1}\left(B_{\varepsilon}\right)\right), v^{-1}\left(S_{\varepsilon}\right), \mathbb{Z}\right)
$$

que avaliado na classe fundamental da homologia do par $\left[v^{-1}\left(B_{\mathcal{\varepsilon}}\right), v^{-1}\left(S_{\mathcal{\varepsilon}}\right)\right]$ dá a obstrução de Euler local

$$
E u_{p}(X)=<E u(\tilde{E}, r),\left[v^{-1}\left(B_{\varepsilon}\right), v^{-1}\left(S_{\varepsilon}\right)\right]>\in \mathbb{Z} .
$$

\subsubsection{Classe de Mather e o isomorfismo $T$}

Seja $X$ uma variedade analítica complexa da variedade suave compacta $M$.

Consideremos o blow up de Nash $\tilde{X} \stackrel{\nu}{\rightarrow} X$ de $X$, o fibrado de Nash $\tilde{T} \stackrel{\pi}{\rightarrow} \tilde{X}$ e as classes de Chern de $\tilde{T}, c^{j}(\tilde{T}) \in H^{2 j}(\tilde{X})$. O homomorfismo de Poincaré

$$
H^{2 n-2 k}(\tilde{X}) \stackrel{\cap[\tilde{X}]}{\longrightarrow} H_{2 k}(\tilde{X})
$$

as carrega em classes de homologia que podem ser levadas na homologia de $X$ via o homomorfismo $v_{*}$ induzido pela projeção.

Definição 4.6. $A$ k-ésima classe de Mather de X é definida como a classe em homologia

$$
c_{k}^{m a}(X):=v_{*}\left(c^{n-k}(\tilde{T}) \cap[\tilde{X}]\right) \in H_{2 k}(X) .
$$

A $k$-ésima classe de Mather de uma função construtível $\alpha=\sum_{i=1}^{n} \alpha_{i} 1_{\left.\right|_{i}}$ é definida como sendo

$$
c_{k}^{m a}(\alpha):=\sum_{i=1}^{n} \alpha_{i} c_{k}^{m a}\left(Y_{i}\right)
$$


Denotemos por $c^{m a}(X)=1+c_{1}^{m a}(X)+\cdots+c_{k}^{m a}(X)+\cdots$ a classe total de Mather de $X$. Analogamente, para $c^{m a}(\alpha)$.

Estas classes não satisfazem os axiomas da conjectura de Deligne-Grothendieck. No entanto, a classe de Mather é um ingrediente fundamental na definição dada por MacPherson. Para solucionar este problema, Macpherson define um isomorfismo $T$ do conjunto dos ciclos algébricos de $V$ em $F(V)$ da seguinte forma:

$$
T\left(\sum_{i \in I, \#(I)<\infty} n_{i} V_{i}\right)(p)=\sum_{i \in I, \#(I)<\infty} n_{i} E u_{p}\left(V_{i}\right), \forall p \in V
$$

Agora sim, obtemos classes $\left(c^{m a} \circ T^{-1}\right)\left(1_{V}\right)$ que satisfazem os axiomas da conjectura de Deligne-Grothendieck.

Definição 4.7. A classe total de MacPherson de uma função construtível $\alpha=\sum_{i=1}^{n} \alpha_{i} 1_{\left.\right|_{Y_{i}}}$ é definida como a classe em homologia $c^{M}(\alpha):=\left(c^{m a} \circ T^{-1}\right)(\alpha)$.

A $k$-ésima classe de MacPherson de $\alpha$ é definida $\operatorname{como} c_{k}^{M}(\alpha):=\left(c_{k}^{m a} \circ T^{-1}\right)(\alpha)$.

Definição 4.8. A classe total de MacPherson de uma variedade analítica complexa $X$ é definida como a classe total de MacPherson da função construtivel $1_{\left.\right|_{X}}$, ou seja, $c^{M}(X):=c^{M}\left(1_{\left.\right|_{X}}\right)$.

Em geral, usaremos simplesmente classe de MacPherson para nos referir a sua classe total.

Observação 4.9. Denotemos por $c^{S}$ as classes de Schwartz. As classes de MacPherson $c^{M}$ são as imagens das classes de Schwartz pelo isomorfismo de Alexander

$$
\begin{array}{cl}
H^{n-i}(X) & \rightarrow H_{i}(X) \\
\sigma & \mapsto \sigma \cap[X]
\end{array},
$$

onde s é a dimensão de X.

$\operatorname{Assim} c_{i}^{M}(X)=c_{s-i}^{S}(X) \cap[X]$. Em particular, $c_{s}^{M}(X)=c_{0}^{S}(X) \cap[X]=1 \cap[X]=[X]$.

Usaremos a notação $c^{S M}$ para as classes de Schwartz-MacPherson (em homologia, $c^{S M}=c^{M}$ e em cohomologia, $\left.c^{S M}=c^{S}\right)$. 


\subsection{Classes de Fulton-Johnson}

Sejam $V$ uma interseção completa local de dimensão $n$ contida em uma variedade suave complexa $M$ de dimensão $n+k$. Isto significa que existe um fibrado vetorial holomorfo $N$ de posto $k$ sobre $M$ e uma seção holomorfa $s$ de $N$ tal que $s$ é genericamente transversal a seção zero e $V$ é o conjunto de zeros de $s$.

Observação 4.10. Seja $V_{\text {reg }}$ a parte regular de $V$. A restrição $N_{V_{\text {reg }}}$ coincide com o fibrado normal $N_{V_{\text {reg }}}$ de $V_{\text {reg }}$ em M. (Para detalhes ver [3], Capítulo 5)

Deste modo temos a seguinte sequência exata

$$
0 \rightarrow T V_{\text {reg }} \rightarrow T M_{\left.\right|_{\text {reg }}} \rightarrow N_{V_{\text {reg }}} \rightarrow 0
$$

Definição 4.11. $O$ fibrado tangente virtual de Vé definido como sendo $T M_{\left.\right|_{V}}-N_{\left.\right|_{V}}$, visto como um elemento na $K$-teoria complexa $K U(V)$.

Observação 4.12. Se $V$ não tem singularidades, ou seja, $V=V_{\text {reg, }}$ temos pelo Teorema do Isomorfismo que

$$
N_{\left.\right|_{V}} \simeq T M_{\left.\right|_{V}} / T V
$$

Assim $T V \simeq T M_{\left.\right|_{V}}-N_{\left.\right|_{V}}$, ou seja, o fibrado tangente virtual de $V$ coincide com o fibrado tangente de $V$.

As classes de Fulton-Johnson utilizam o fibrado virtual em substituição ao fibrado tangente, que em geral são distintas das classes de Schwartz-MacPherson. Recordando que a classe de Chern total é invertível no anel de cohomologia temos a seguinte definição.

Definição 4.13. A classe (total) de Fulton-Johnson de $V$ é definida por

$$
c^{F J}(V)=c\left(T M_{\left.\right|_{V}}-N_{\left.\right|_{V}}\right) \cap[V]
$$

onde TM denota o fibrado tangente de $M$ e a classe de Chern de $\left(T M_{\left.\right|_{V}}-N_{\left.\right|_{V}}\right)$ é

$$
c\left(T M_{\left.\right|_{V}}-N_{\left.\right|_{V}}\right)=i^{*}\left(c(T M) \cdot c(N)^{-1}\right) \text { com } i: V \hookrightarrow M \text { a inclusão e } k \in \mathbb{Z} \text {. }
$$




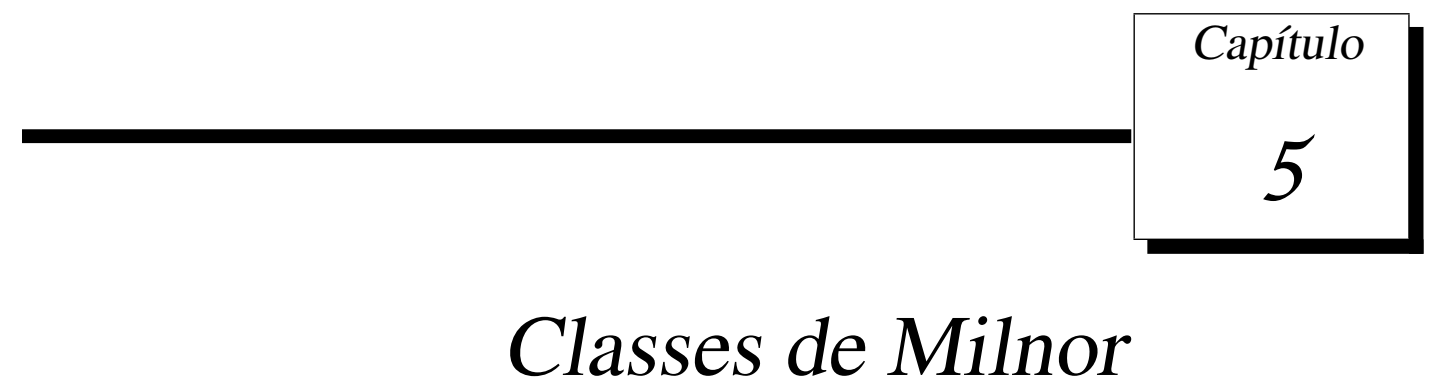

As classes obtidas pela diferença entre as duas generalizações das classes de Chern no caso singular descritas no capítulo anterior, classes de Fulton-Johnson e classes de SchwartzMacPherson, têm sido investigadas por vários pesquisadores. Elas são chamadas de classes de Milnor, que levam este nome pois no caso de singularidade isolada somente a classe de dimensão zero é não nula e esta é a soma dos números de Milnor dos pontos singulares da variedade.

Neste capítulo vamos descrever estas classes no caso de hipersuperfíces em variedades complexas compactas e, usando a descrição via especialização dada por Parusinski e Pragacz, demonstramos primeiro alguns resultados conhecidos, porém ao nosso ver, de maneira mais simples e direta.

Este estudo sobre um outro ponto de vista nos permitiu expressar na Proposição 5.6 a classe de Milnor de dimensão máxima como uma soma, sobre os estratos de uma estratificação de Whitney que estão contidos no conjunto singular, em termos do número de Milnor transversal de cada estrato. Esta é uma generalização de um resultado conhecido de Brasselet, Lehmann, Seade e Suwa em [4] que é válido somente quando o conjunto singular é não singular. Além disso, expressamos o número de Milnor transversal como o último número de Lê associado a esta hipersuperfície.

Ressaltamos que os resultados conhecidos, que são mencionados neste capítulo e cuja referência é [4], são válidos não somente para hipersuperfíces mas para o caso de intersecções completas locais.

Além disso, demos uma interpretação geométrica do número de Milnor de Parusinki (Teorema 5.9) sem utilizar a especialização. 
Durante este capítulo, denotamos por $M$ uma variedade complexa compacta de dimensão $n$ e por $Z$ uma hipersuperfície de $M$.

Definição 5.1. A classe total de Milnor de Z é definida por

$$
\mathscr{M}(Z):=(-1)^{n-1}\left(c^{F J}(Z)-c^{S M}(Z)\right)
$$

A $k$-ésima classe de Milnor é a diferença da $k$-ésima classe de Fulton-Johnson de $Z$ pela $k$-ésima classe de Schwartz-MacPherson de $Z$.

Em geral, usaremos simplesmente classe de Milnor para nos referir a sua classe total.

Exemplo 5.2. Suponha que o conjunto singular de Z é um número finito de pontos $x_{1}, \cdots, x_{r}$. Denotemos por $\mu_{x_{i}}$ o número de Milnor de $Z$ em $x_{i}$. Então $\mathscr{M}_{0}(Z)=\sum_{i=1}^{r} \mu_{x_{i}}\left[x_{i}\right] \in H_{0}(Z)$.

Para a demonstração ver [4], Teorema 5.2.

Seja $V$ uma variedade de dimensão $n$ que é uma intersecção completa local em uma variedade suave complexa $M$ de dimensão $n+k$. A seguir descrevemos um resultado bem conhecido que garante a existência de um fibrado holomorfo de posto $k$ sobre $M$ onde $V$ é o conjunto de zeros de uma seção holomorfa deste fibrado.

Vamos enunciá-lo para o caso de hipersuperfícies. A demonstração a seguir segue de maneira análoga ao Exemplo 5.11 do Capítulo I de [28].

Proposição 5.3. Existe um fibrado vetorial de linha sobre $M$ tal que $Z$ é o conjunto de zeros de uma seção holomorfa desse fibrado.

Demonstração. Como $Z$ é uma hipersuperfície de $M$, dada uma cobertura aberta $\left\{U_{i}\right\}_{i=1}^{r}$ de $M$, existem funções holomorfas $f_{i}: U_{i} \rightarrow \mathbb{C}$ tais que $U_{i} \cup Z=f_{i}^{-1}(0)$ e $f_{\left.i\right|_{U_{i} \cap U_{j}}}=f_{\left.\right|_{U_{i} \cap U_{j}}}, \forall i, j$.

Essas funções definem um fibrado vetorial de linha sobre $M$ da seguinte forma:

Considere a relação: Para $(p, c) \in U_{i} \times \mathbb{C} \mathrm{e}(q, d) \in U_{j} \times \mathbb{C},(p, c) \sim(q, d) \Rightarrow\left\{\begin{array}{l}p=q \\ c=f_{i}(p) d\end{array}\right.$

É fácil ver que essa é uma relação de equivalência.

Denotemos $E=\biguplus U_{i} \times \mathbb{C} / \sim$, onde $[(p, c)]$ é um elemento de $E$. Seja $\pi: E \rightarrow X$, com $\pi([(p, c)]):=p$. Assim $\pi^{-1}\left(U_{i}\right)=\left\{[(p, c)], p \in U_{i}\right\}$. Definimos 


$$
\begin{aligned}
\psi_{i}: \pi^{-1}\left(U_{i}\right) & \rightarrow U_{i} \times \mathbb{C} \\
{[(p, c)] } & \mapsto(p, c)
\end{aligned}
$$

Assim, para cada $(p, c) \in U_{i} \times \mathbb{C}$,

$$
\psi_{j} \circ \psi_{i}^{-1}(p, c)=\psi_{j}([(p, c)])=\psi_{j}\left(\left[\left(p, f_{i}(p) c\right)\right]\right)=\left(p, f_{i}(p) c\right) .
$$

Denotemos por $L$ esse fibrado, ou seja, $L=(E, X ; \pi)$. Considere $s$ uma seção de $L$ tal que

$$
\begin{aligned}
s: U_{i} & \stackrel{\sim}{\rightarrow} U_{i} \times \mathbb{C} \\
p & \mapsto\left(p, f_{i}(p)\right)
\end{aligned}
$$

Temos que $s$ é uma seção holomorfa pois $f_{i}$ é holomorfa $\forall i=1, \cdots, r$ e $Z$ é o conjunto de zeros de $s$.

O seguinte resultado é também bem conhecido (Ver [4], Teorema 5.2). Usando um resultado de Parusinski-Pragacz segue uma prova de um diferente ponto de vista.

Proposição 5.4. As classes de Milnor têm suporte em Sing(Z). Daí estas classes são todas nulas em dimensões acima da dimensão de $\operatorname{Sing}(Z)$.

Demonstração. Seja $\mathscr{S}=\{S\}$ uma estratificação de Whitney de $Z$ com estratos conexos e Sing $Z$ uma união de estratos.

Em [2], Parusinski e Pragacz descrevem a classe de Milnor como segue:

$$
\mathscr{M}(Z):=\sum_{S \in \mathscr{B}} \alpha_{S}\left(c\left(L_{\mid Z}\right)^{-1} \cap\left(i_{\bar{S}, Z}\right)_{*} c^{S M}(\bar{S})\right)
$$

onde $\alpha_{S}=\mu_{S}-\sum_{S^{\prime} \neq S, \bar{S}^{\prime} \supset S} \alpha_{S^{\prime}} \operatorname{com} \mu_{S}=(-1)^{n-1}\left(\chi\left(F_{x}\right)-1\right)$, para algum $x \in S, F_{x}$ denota a característica de Euler da fibra da Milnor de $Z$ em $x$ e $i_{\bar{S}, Z}: \bar{S} \hookrightarrow Z$ denota a inclusão.

Se $x$ é um ponto regular de $Z$, então $\chi\left(F_{x}\right)=1$ e $\mu_{S}=0$. Daí

$$
\mathscr{M}(Z)=\sum_{S \in \operatorname{Sing} Z} \alpha_{S}\left(c\left(L_{\mid Z}\right)^{-1} \cap\left(i_{\bar{S}, Z}\right)_{*} c^{S M}(\bar{S})\right)
$$

Como $\operatorname{dim} \bar{S} \leq \operatorname{dim}(\operatorname{Sing} Z), c_{i}^{S M}(S)=0$ se $i>\operatorname{dim}(\operatorname{Sing} Z)$. Portanto, $\mathscr{M}_{i}(Z)=0$ if $i>$ $\operatorname{dim}(\operatorname{Sing} Z)$. 


\subsection{Classe de Milnor de dimensão máxima}

Dada $\{W\}$ as componentes conexas de $\operatorname{Sing} Z$ e denote por $s$ a dimensão de $\operatorname{Sing} Z$, temos o seguinte resultado conhecido:

Proposição 5.5. Se Sing $Z$ é não-singular, $\mathscr{M}_{s}(Z)=\sum_{\operatorname{dim} W=s}(-1)^{s} \mu(W) \cdot[W], \operatorname{com} \mu(W) o$ número de Milnor transverso de W.

Para detalhes ver [4], Teorema 5.2 e Corolario 5.13.

Consideremos agora o caso onde $\operatorname{Sing} Z$ é qualquer, ou seja, possivelmente singular. Além disso, vamos assumir que existe uma seção $s^{\prime} \in H^{0}(X, L)$ tal que a variedade $Z^{\prime}$ dos zeros de $s^{\prime}$ é suave e transversal a todos os estratos da estratificação de Whitney $\mathscr{B}$.

Teorema 5.6. Na notação acima,

$$
\mathscr{M}_{S}(Z)=\sum_{S \in \operatorname{Sing}} \underset{Z, \operatorname{dim} S=s}{ }(-1)^{s} \mu(S)[\bar{S}]=\sum_{S \in \operatorname{Sing}} \underset{Z, \operatorname{dim} S=s}{ }(-1)^{s} \lambda_{S}^{s}[\bar{S}] .
$$

Demonstração. Com esta nova condição, Parusinski e Pragacz (ver [2]) dão outra descrição da classe de Milnor (via a especialização acima),

$$
\mathscr{M}(Z):=\sum_{S \in \mathscr{B}} \alpha_{S}\left[\left(i_{\bar{S}, Z}\right)_{*} c^{M}(\bar{S})-\left(i_{\bar{S} \cap Z^{\prime}, Z}\right)_{*} c^{M}\left(\bar{S} \cap Z^{\prime}\right)\right]
$$

onde $\alpha_{S}=\mu_{S}-\sum_{S^{\prime} \neq S, \bar{S}^{\prime} \supset S} \alpha_{S^{\prime}} \operatorname{com} \mu_{S}=(-1)^{n-1}\left(\chi\left(F_{x}\right)-1\right)$, para algum $x \in S, F_{x}=F_{f_{j}, x}$ denota a característica de Euler da fibra da Milnor de $Z$ em $x \in U_{j} \operatorname{com} f_{j}$ a função de definição de $Z$ definida em $U_{j}$ e $i_{\bar{S}, Z}: \bar{S} \hookrightarrow Z$ denota a inclusão.

Agora temos que $\bar{S}=\bigcup_{k} S_{k}$ e, com isso, $\bar{S} \cap Z^{\prime}=\bigcup_{k} S_{k} \cap Z^{\prime}$. Como $Z^{\prime}$ é transversal a cada estrato, $\operatorname{dim} S_{k} \cap Z^{\prime}=\operatorname{dim} S_{k}-1 . \operatorname{Se} \operatorname{dim} S=s$, então $\operatorname{dim} \bar{S} \cap Z^{\prime}=$ máximo $_{k}\left\{S_{k} \cap Z^{\prime}\right\}=s-1$. Portanto, $c_{s}^{M}\left(\bar{S} \cap Z^{\prime}\right)=0$.

Daí, usando a observação 4.9,

$$
\mathscr{M}_{s}(Z)=\sum_{S \in \operatorname{Sing}} \sum_{Z, \operatorname{dim} S=s} \mu_{S}\left(i_{\bar{S}, Z}\right)_{*} c_{s}^{M}(\bar{S})=\sum_{S \in \operatorname{Sing}} \sum_{Z, \operatorname{dim} S=s} \mu_{S}[\bar{S}] .
$$


Afirmação. $\mu_{S}=(-1)^{s} \mu(S)$, onde $\mu(S)$ é o número de Milnor transverso de $S$.

O número de Milnor transverso de $S$ é definido como sendo o número de Milnor $\mu\left(\left(f_{j}\right)_{\left.\right|_{T_{x}}}\right)$ para algum $x \in U_{j}$ em $S, f_{j}$ é uma das funções de definição de $Z$ definida em $U_{j}$ e $T_{x}$ é um plano genérico de dimensão complementar a $\operatorname{Sing} Z$ em $M$.

Como $S$ tem a mesma dimensão do conjunto singular, $T_{x}$ é genérico e usando o fato de que todo conjunto Whitney estratificado topologicamente trivial localmente temos que existe um homeomorfismo local $Z, x \rightarrow S, x \times N, x$, com $N=T_{x} \cap Z$. Além disto, como toda função holomorfa satisfaz a condição de Thom, podemos concluir que $\chi\left(F_{f_{j}, x}\right)=\chi\left(F_{\left(\left.f_{j}\right|_{\left.\right|_{x}}, x\right.}\right)$.

Pelo Teorema da Fibração de Milnor, $\chi\left(F_{\left(f_{j}\right)_{\left.\right|_{x}}, x}\right)=1+(-1)^{n-s+1} \mu\left(\left(f_{j}\right)_{\left.\right|_{T_{x}}}\right)$.

Assim, $\mu_{S}=(-1)^{n-1}\left(\chi\left(F_{f_{j}, x}\right)-1\right)=(-1)^{s} \mu\left(\left(f_{j}\right)_{\left.\right|_{T_{x}}}\right)=(-1)^{s} \mu(S)$.

Aliás, seja $s$ a dimensão do conjunto crítico de um germe $f$. Como conjunto, o ciclo de Lê s-dimensional é exatamente a união das componentes s-dimensionais do conjunto crítico.

Por definição da estrutura de ciclo, em um ponto genérico em $\Lambda_{f, z}^{s}$, a estrutura é dada pelo anulamento de $\partial f / \partial z_{s}, \ldots \partial f / \partial z_{n}$.

A propriedade fundamental de teoria de intersecção é que podemos perturbar as equações e contar intersecções com multiplicidades dentro de uma pequena bola.

Deste modo, $\lambda_{f, z}^{s}(0)=\left(V\left(z_{0}, \ldots, z_{s-1}\right) \cdot \Lambda_{f, z}^{s}\right)_{0}$ é iqual a uma soma sobre todos os pontos, dentro de uma pequena bola centrada na origem, de todas os números de intersecções de $V\left(z_{0}-a_{0}, \ldots, z_{s-1}-a_{s-1}\right) \operatorname{com} \Lambda_{f, z}^{s}$, onde os $a_{0}, \ldots, a_{s-1}$ são pequenos comparados com o tamanho da bola.

Isto dá $\eta_{v}$ pontos em cada componente s-dimensional $v$, e cada um destes pontos é contado com a multiplicidade do ideal gerado por $\left(z_{0}-a_{0}, \ldots, z_{s-1}-a_{s-1}, \partial f / \partial z_{s}, \ldots \partial f / \partial z_{n}\right)$. Este é exatamente o número de Milnor de $f$ restrita a $V\left(z_{0}-a_{0}, \ldots, z_{s-1}-a_{s-1}\right)$, isto é, o número de Milnor transverso em um ponto genérico. (Para detalhes ver [17], 1.19.)

Usando que o número de Lê genérico $\lambda_{g_{i}}^{s}$, com $g_{i}=f_{i} \circ h_{i}^{-1}$, é constante ao longo de cada estrato de qualquer estratificação de Whitney de $V\left(g_{i}\right)$ ([17], Teorema 10.19.) e que os estratos são conexos, pelo Lema 2.12 temos um número $\lambda_{S}^{s}$ constante ao longo de cada estrato $S$ da estratificação de Whitney $\mathscr{B}$ de $Z$, onde $\lambda_{S}^{s}=\lambda_{g_{i}}^{s}(x)$ para algum $i$ tal que $S \cap U_{i} \neq$ e $x \in S$.

Daí, $\lambda_{S}^{s}$ é o número de Milnor transverso em $S$. 


$$
\text { Portanto, } \mathscr{M}_{S}(Z)=\sum_{S \in \text { Sing }} \underset{Z, \operatorname{dim} S=s}{ }(-1)^{s} \mu_{S}[\bar{S}]=\sum_{S \in \operatorname{Sing}} \underset{Z, \operatorname{dim} S=s}{ }(-1)^{s} \lambda_{S}^{s}[\bar{S}] .
$$

Observação 5.7. Acreditamos que o teorema anterior é verdadeiro sem a hipótese de especialização, porém em nossa demonstração esta hipótese é utilizada. Deste modo, uma questão interessante é descobrir uma demonstração deste resultado sem a especialização.

\subsection{Classe de Milnor de dimensão mínima}

A primeira classe de Milnor $\mathscr{M}_{0}(Z) \in H_{0}(Z) \simeq \mathbb{Z}$ coincide com o número definido por Parusinski (Teorema 6.2 de [4]), chamado número de Milnor de Parusinski e denotado por $\mu_{P}(Z)$, que é uma generalização do número de Milnor no caso de singularidades não isoladas (Proposição 1.4 de [23]). Quando temos a hipótese de especialização esta igualdade também pode ser vista usando o Teorema 0.2 de [25] e Proposição 7 de [24], que é feita a partir da caracterização das classes de Milnor por Parusinski e Pragacz, como anteriormente mencionadas.

Dentre as várias interpretações deste número, uma delas é que o número de Milnor de Parusinski $\mu_{P}(Z)$ pode ser visto da seguinte maneira ([23], Proposição 1.6):

$$
\mu_{P}(Z)=(-1)^{n} \chi(Z)+<c_{n}\left(T^{*^{\prime}} M \otimes L\right),[M]>-(-1)^{n} \chi(M) .
$$

Como corolário temos que

Corolário 5.8. ([23], 1.7) Se as hipersuperfícies $Z$ e Z' são zeros de seções de um mesmo fibrado de linha sobre uma variedade complexa compacta, então

$$
\mu_{P}(Z)-\mu_{P}\left(Z^{\prime}\right)=(-1)^{n}\left(\chi(Z)-\chi\left(Z^{\prime}\right)\right)
$$

O próximo resultado dá uma interpretação geométrica do número de Parusisnki sem a especialização, que é consequência das fórmulas de Lê-Iomdine generalizadas (Teorema 3.5) e do Corolário 5.8.

Teorema 5.9. Dada uma hipersuperfície $Z$ numa variedade compacta $M$, existe uma hipersuperfície $Z^{\prime}$ com singularidades isoladas $\left\{p_{1}, \cdots, p_{l}\right\}$ tal que

$$
\mu_{P}(Z)=\sum_{j} \sum_{k}\left(\prod_{n=0}^{k-1}\left(j_{n, i}-1\right) \lambda_{\left(f_{i}\right)_{j}}^{k}\left(p_{j}\right)\right)+(-1)^{n}\left(\chi(Z)-\chi\left(Z^{\prime}\right)\right)
$$


onde $\left\{f_{i}\right\}$ são as funções de definição de $Z$ e $\lambda_{\left(f_{i}\right)_{j}}^{k}\left(p_{j}\right)$ são os k-ésimos números de Lê de $f_{i}$ que têm $p_{j}$ como ponto singular.

Demonstração. Como $Z$ é uma hipersuperfície em uma variedade complexa compacta $M$, existem uma cobertura aberta e finita $\left\{U_{i}\right\}_{i=1}^{r}$ de $Z$ e funções holomorfas $f_{i}: U_{i} \rightarrow \mathbb{C}$ tais que $U_{i} \cup Z=f_{i}^{-1}(0)$ e $f_{\left.i\right|_{U_{i} \cap U_{j}}}=f_{\left.\right|_{U_{i} \cap U_{j}}}, \forall i, j$.

Para não trabalhar com as cartas coordenadas e carregar a notação, vamos supor que $U_{i}$ é um aberto de $\mathbb{C}^{n+1}$.

Podemos definir em cada $U_{i}$ um sistema linear genérico de coordenadas tal que os números de Lê $\lambda_{f_{i}}^{j}(p)$ estão definidos para todo $p \in U_{i}$ e $j \leq \operatorname{dim}\left(\Sigma f_{i}\right)$. (Teorema 2.9).

Como esta cobertura é finita, existe um sistema linear genérico de coordenadas $z=\left(z_{0}, \ldots, z_{n}\right)$ tal que $\lambda_{f_{i}}^{j}(p)$ está definido para todo $p \in U_{i}, i=1, \cdots, r$ e $j \leq s \operatorname{com} s=\operatorname{dim}(\operatorname{Sing} Z)$.

Agora, para cada função $f_{i}$ da definição de $Z$, aplicando o Teorema das fórmulas de LêIomdine generalizadas (Teorema 3.5), existem $a_{k} \in \mathbb{C}^{*}$ e $j_{k, i} \in \mathbb{Z} \operatorname{com} k=0, \cdots, s-1$ tais que

$$
f_{i}+a_{0} z_{0}^{j_{0, i}}+\cdots+a_{s-1} z_{s-1}^{j_{s-1, i}}
$$

tem singularidades isoladas.

Considerando $j_{i}=\max _{0 \leq k \leq s-1} j_{k, i}$ podemos definir uma seção $s$ em $L$ da seguinte forma

$$
s_{\left.\right|_{i}} \simeq f_{i}+a_{0} z_{0}^{j_{0}}+\cdots+a_{s-1} z_{s-1}^{j_{s-1}}:=g_{i}
$$

Seja $Z^{\prime}$ o conjunto de zeros de $s$. Assim, usando o Corolário 5.8,

$$
\mu_{P}(Z)=\mu_{P}\left(Z^{\prime}\right)+(-1)^{n}\left(\chi(Z)-\chi\left(Z^{\prime}\right)\right)
$$

Pela construção, as singularidades de $Z^{\prime}$ são isoladas, digamos $\left\{p_{1}, \cdots, p_{l}\right\}$.

Denotemos por $\left(g_{i}\right)_{j}$ a aplicação $g_{i}$ (ou melhor dizendo, uma das aplicações $g_{i}^{\prime}$ s) que tem $p_{j}$ como ponto singular.

Assim,

$$
\mu_{P}\left(Z^{\prime}\right)=\sum_{j} \mu_{P}\left(Z^{\prime}, p_{j}\right)=\sum_{j} \mu_{M}\left(\left(g_{i}\right)_{j}, p_{j}\right)
$$

onde aqui $\mu_{P}$ denota o número de Milnor de Parusinski e $\mu_{M}$ o número de Milnor. 
Para cada $j \in\{1, \cdots, l\}$, obtemos do Teorema das fórmulas de Lê-Iomdine generalizadas (Teorema 3.5) que $\mu_{M}\left(\left(g_{i}\right)_{j}, p_{j}\right)=\sum_{k}\left(\prod_{n=0}^{k-1}\left(j_{n, i}-1\right) \lambda_{\left(f_{i}\right)_{j}}^{k}\left(p_{j}\right)\right)$.

Portanto,

$$
\mu_{P}(Z)=\sum_{j} \sum_{k}\left(\prod_{n=0}^{k-1}\left(j_{n, i}-1\right) \lambda_{\left(f_{i}\right)_{j}}^{k}\left(p_{j}\right)\right)+(-1)^{n}\left(\chi(Z)-\chi\left(Z^{\prime}\right)\right)
$$




\section{Referências Bibliográficas}

[1] M. F. Atiyah and I. G. Macdonald, Introduction to commutative algebra, Addison-Wesley series in Math. (Addison-Wesley Publishing Company, Massachusetts, 1969).

[2] J.-P. Brasselet and M.H. Schwartz, Sur les classes de Chern d'un ensemble analytique complexe, Astérisque 82-83 (1981), 93-147.

[3] J.-P. Brasselet, J. Seade and T. Suwa, Vector Fields on Singular Varieties, Lect. Notes Math. 1987. (Springer, 2009).

[4] J.P. Brasselet, D. Lehmann, J. Seade and T. Suwa, Milnor classes of local complete intersections, Transactions of the AMS. 354 (2001), 1351-1371.

[5] S. S. Chern, Characteristic classes of hermitian manifold, Annals of Math. 47 (1945), 85-121.

[6] D. Eisenbud and J. Harris, Schemes: the language of modern algebraic geometry (Wadsworth \& Brooks/Cole, California, 1992).

[7] W. Fulton, Intersection theory, Erg. der Math. und ihrer Grenz.: 3, Folge, Bd. 2 (SpringerVerlag, Berlim; New York, 1984).

[8] W. Fulton and K. Johnson, Canonical classes on singular varieties, Manuscripta Math. 32 (1980), 381-389.

[9] T. Gaffney and R. Gassler, Segre numbers and hypersurface singularities, J. Algebraic Geometric 08 (1999), 695-736.

[10] C. G. Gibson, Singular points of smooth mappings, Research Notes Math. 25 (Pitman Publishing, London; San Francisco, 1979). 
[11] C. G. Gibson, K. Wirthmüller, A. Plessis and E.J. Looijenga, Topological stability of smooth mappins, Lect. Notes Math. 552. (Springer-Verlag, Berlim; New York, 1976).

[12] P. Griffiths and J. Harris, Principles of algebraic geometry, Pure and Applied Math. (Wiley, New York, 1978).

[13] D. T. Lê and B. Teissier, Variétés polaires locales et classes de Chern de variétés singulères, Annals of Math. 114 (1981), 457-491.

[14] E. L. Lima, Variedades diferenciáveis, Monografias de Matemática 15 (IMPA, Rio de Janeiro 1973).

[15] E. L. Lima, Homologia Básica, Projeto Euclides (IMPA, Rio de Janeiro, 2009).

[16] R. MacPherson, Chern classes for singular algebraic varieties, Annals of Math. 100, no. 2 (1974), 423-432.

[17] D. Massey, Lê cycles and hypersurface singularities, Lect. Notes Math. 1615. (Springer, Berlim, 1995).

[18] J. Milnor and P. Orlik, Isolated singularities defined by weighted homogeneous polynomials, Topology 9 (1970), 385-393.

[19] J. Milnor, Singular points of complex hypersurfaces, Annals of Math. Studies 61 (Princeton University Press, Princeton, N.J., 1968).

[20] J. Milnor, Topology from the differentiable viewpoint (Univ. Press of Virginia, Charlottesville, 1965).

[21] J. Milnor, Characteristic classes, Annals of Math. Studies 76 (Princeton University Press, Princeton, N.J., 1974).

[22] J. Munkres, Elementary Differential Topology, Annals of Math. Studies 54 (Princeton University Press, Princeton, N.J., 1966).

[23] A. Parusinski, Generalization of the Milnor number, Math. Ann. 281 (1988), 247-254. 
[24] A. Parusinski and P. Pragacz, A formula for the Euler Characteristic of singular hypersurfaces, J. Algebraic Geometry 4 (1995), 337-351.

[25] A. Parusinski and P. Pragacz, Characteristic classes of hypersurfaces and characteristic cycles, J. Algebraic Geometry 10 (2001), 63-79 .

[26] M.H. Schwartz, Classes caractéristiques définis par une stratification d'une variété analytique complexe, CRAS, 260 (1965), 3262-3264 and 3535-3537.

[27] Y. T. Siu and G. Trautmann, Gap-sheaves and extension of coherent analytic subsheaves, Lect. Notes Math. 172 (Springer-Verlag, Berlim; New York, 1971).

[28] T. Suwa, Indices of vector fields and residues of singular holomorphic foliations, Actualités Mathématiques (Hermann, Paris, 1998).

[29] B. Teissier, Variétés polaires. II. Multiplicités polaires, sections planes et conditions de Whitney, Lecture Notes in Math. 961 (Springer, Berlim, 1982).

[30] O. Zariski and P. Samuel, Commutative algebra, Vol. 1 (Van Nostrand, Princeton, 1958).

[31] H. Whitney, Tangents to an analytic variety, Ann. Math. 81, no. 3 (1965), 496-549. 